\title{
Study of doubly heavy tetraquarks in lattice QCD
}

\author{
Parikshit Junnarkar, ${ }^{1, *}$ Nilmani Mathur, ${ }^{1, \dagger}$ and M. Padmanath ${ }^{2}$ \\ ${ }^{1}$ Department of Theoretical Physics, Tata Institute of Fundamental Research, \\ 1 Homi Bhabha Road, Mumbai 400005, India \\ ${ }^{2}$ Institüt für Theoretische Physik, Universität Regensburg, D-93040 Regensburg, Germany
}

(Received 22 November 2018; published 20 February 2019)

\begin{abstract}
We present the results of a lattice calculation of tetraquark states with quark contents $q_{1} q_{2} \bar{Q} \bar{Q}, q_{1}, q_{2} \subset$ $u, d, s, c$ and $Q \equiv b, c$ in both spin-0 $(J=0)$ and spin-1 $(J=1)$ sectors. This calculation is performed on three dynamical $N_{f}=2+1+1$ highly improved staggered quark ensembles at lattice spacings of about $0.12,0.09$, and $0.06 \mathrm{fm}$. We use the overlap action for light to charm quarks, while a nonrelativistic action with nonperturbatively improved coefficients with terms up to $\mathcal{O}\left(\alpha_{s} v^{4}\right)$ is employed for the bottom quark. While considering charm or bottom quarks as heavy, we calculate the energy levels of various four-quark configurations with light quark masses ranging from the physical strange quark mass to that of the corresponding physical pion mass. This enables us to explore the quark mass dependence of the extracted four-quark energy levels over a wide range of quark masses. The results of the spin-1 states show the presence of ground state energy levels which are below their respective thresholds for all the light flavor combinations. Further, we identify a trend that the energy splittings, defined as the energy difference between the ground state energy levels and their respective thresholds, increase with decreasing the light quark masses and are maximum at the physical point for all the spin- 1 states. The rate of increase is, however, dependent on the light quark configuration of the particular spin-1 state. We also present a study of hadron mass relations involving tetraquarks, baryons, and mesons arising in the limit of infinitely heavy quarks and find that these relations are more compatible with the heavy quark limit in the bottom sector but deviate substantially in the charm sector. The ground state spectra of the spin-0 tetraquark states with various flavor combinations are seen to lie above their respective thresholds.
\end{abstract}

DOI: $10.1103 /$ PhysRevD.99.034507

\section{INTRODUCTION}

The past decade and a half has seen a remarkable number of discoveries in heavy hadrons. These new findings not only include regular mesons [1-7] and baryons [8,9] but also involve exotic hadrons like tetra- [10-12] and pentaquarks [13], while the structures of many are still puzzling (like many of the so-called $X, Y$, and $Z$ states) [14-22]. These hadrons, in particular, the multiquark states, are reshaping our understanding of bound states and are providing new insights into the dynamics of strong interactions at multiple scales. Among the most notable multiquarks hadrons, $Z_{b}(10630)$ and $Z_{b}^{\prime}(10650)$ were discovered first [12], followed by $Z_{c}(4430)$ [10-12] and then $P_{c}$ pentaquarks [13]. Naturally, these discoveries have

\footnotetext{
"parikshit@theory.tifr.res.in

nilmani@theory.tifr.res.in
}

Published by the American Physical Society under the terms of the Creative Commons Attribution 4.0 International license. Further distribution of this work must maintain attribution to the author(s) and the published article's title, journal citation, and DOI. Funded by SCOAP . kicked off a flurry of activities in heavy hadron physics, both theoretically and experimentally, and there is a real prospect of discovering more exotic hadrons, particularly with one or more bottom quark contents at various laboratories [23-26]. The current status of these new discoveries, particularly on exotics, is provided in various recent review articles [18-20,27-29].

Theoretical studies of exotic hadrons are not new. Among the exotics, perhaps tetraquarks are the most studied states. Historically, they were introduced by Jaffe [30] as color neutral states of diquarks and antidiquarks ${ }^{1}$ in the context of describing light scalar mesons as tetraquarks and later for exotic spectroscopy [31,32]. Subsequently, the diquark picture of tetraquarks was investigated in detail by many authors through various models [18-20,27,28]. Phenomenologically, a four-quark state can also be modeled as molecules [33,34], hadroquarkonia [35,36], and

\footnotetext{
${ }^{1} \mathrm{~A}$ diquark can be interpreted as a compact colored object inside a hadron and is made out of two quarks (or antiquarks) in the $\overline{\mathbf{3}}(\mathbf{3})$ or $\mathbf{6}(\overline{\mathbf{6}})$ irrep of SU(3) and can have spin 0 (scalar) or spin 1 (vector). With this model, one can build rich phenomenology for mesons, baryons, as well as multiquark states.
} 
also as threshold cusps [37,38], depending on how the four quarks interact mutually.

Though these models are effective with varying degrees in describing these states, it is essential to have a first principles description of these strongly interacting hadrons. Lattice QCD, being a first principles nonperturbative method, ideally provides such an avenue to investigate these states comprehensively. The success of lattice QCD, however, is still limited for these exotic states for multiple reasons. First, almost all such states that are observed lie very close to their threshold energy levels. Though substantial progress has been made for resolving close-by states, it is essential to use novel techniques like distillation [39] that allows for the construction of a large set of operators with the desired overlap onto the ground state which can then be computed using the variational principle $[40,41]$. Second, to identify a resonance state unambiguously from its noninteracting thresholds, one has to perform the rigorous finite volume analysis [41] of the discrete spectrum on multiple volumes and/or multiple momentum frames. Moreover, these heavy hardons are very much susceptible to discretization error, and a precise statement cannot be made unless one takes a controlled continuum limit of the results obtained at finite lattice spacings. All these issues amount to a very large computationally intensive calculation, which presumably will be carried out in the future but currently is beyond the scope of any lattice group.

Current lattice QCD methods with available computational resources can, however, be a useful tool for studying hadrons which are far below their strong decay thresholds. For example, taking advantage of these methods and available computational resources, one can study the deeply bound multiquark states to investigate whether such state exist in nature. One can employ lattice methodology for a systematic search for these states using various spin and flavor combinations of interpolating operators and then, dialing the quark masses, spanning over a wide range, can study the onset of a stable state with a large binding energy. In fact, it was already speculated several years ago that there may exist deeply bound tetraquark states in the heavy quark limit. Using one pion exchange between the ground state $Q \bar{q}$ mesons, Manohar and Wise showed that QCD contains stable (under strong interactions) four-quark $Q Q \bar{q} \bar{q}$ hadronic states in the infinite quark mass limit, and for the bottom quark, this binding could well be sufficiently large [42].

The heavy tetraquarks were also studied recently using heavy quark effective theory [24,43], quark models [23,4450], QCD sum rules [51-53], and large $N_{c}$ calculations $[54-56] .^{2}$ The proposed doubly bottom tetraquark state and

\footnotetext{
${ }^{2}$ There are many model calculations on tetraquarks, and for a detailed reference list, readers may want to see review articles [18-20,27-29].
}

its isospin cousins are believed to be strong interaction stable states with relatively long lifetimes. Recently, lattice QCD calculations [25,57] and a lattice-QCD-potential based study [58-60] also identified a particular exotic flavor-spin combination of two bottom quarks, namely $u d \bar{b} \bar{b}$, with a prediction of a deeply bound state which lies below its noninteracting two-meson threshold. It is thus quite crucial to investigate such and similar states using a detailed lattice QCD study by incorporating various heavy and light flavor combinations along with different spin combinations and at multiple lattice spacings.

In this work, we carry out such a calculation in which we use both the charm and bottom as heavy quarks and then vary the light quark masses from the strange quark mass to the corresponding lower pion masses leading to various tetraquark states: $q_{1} q_{2} \bar{Q} \bar{Q}, q_{1}, q_{2} \subset u, d, s, c$ and $Q \equiv b, c$ with both spin $0(J=0)$ and spin $1(J=1)$. These are computed at three lattice spacings of approximately 0.12 , 0.09 , and $0.06 \mathrm{fm}$ to investigate the discretization effects on these heavy hadrons. We use the relativistic overlap action, for light to charm quarks, while a nonrelativistic action with nonperturbatively improved coefficients with terms up to $\mathcal{O}\left(\alpha_{s} v^{4}\right)$ is employed for the bottom quark. Our results for the spin-1 tetraquarks indicate the presence of energy levels below the respective thresholds for all light flavor combinations with doubly heavy, in particular, for doubly bottom, quarks. The results for spin-0 tetraquarks, which are the flavor symmetric cousin states of the spin-1 counterparts, however, indicate the respective energy levels are above their lowest strong decay two-meson thresholds. In addition to computing the ground state spectra, we also present a lattice study of the hadron mass relations between tetraquarks, heavy baryons, and mesons arising from the heavy quark symmetry. In the future, we will incorporate also the finite volume study so that more quantitative conclusions about the pole structures of these tetraquark states can be made, particularly for the near-threshold states.

The paper is organized as follows. In Sec. II, we elaborate the lattice setup, actions employed, and quark mass combinations that we use for this work. Section III provides details of the tetraquark operators and the flavorspin combinations that we employ in this work. In Sec. IV, with the details of analysis method, we present our results, first for the spin-1 sector followed by the spin-0 sector. Finite volume effects on our results are discussed thereafter. A discussion on the hadron mass relations with the heavy quark symmetry follows afterward. Finally, conclusions from this work are discussed in Sec. V.

\section{LATTICE SETUP}

We perform this calculation on three dynamical $2+1+1$ flavors lattice ensembles generated by the MILC Collaboration [61]. These ensembles, with lattice sizes $24^{3} \times 64,32^{3} \times 96$ and $48^{3} \times 144$, at gauge couplings 
$10 / g^{2}=6.00,6.30$ and 6.72 , respectively, were generated with the highly improved staggered quark (HISQ) action and with the one-loop, tadpole improved Symanzik gauge action with coefficients corrected through $\mathcal{O}\left(\alpha_{s} a^{2}, n_{f} \alpha_{s} a^{2}\right)$ [62]. The masses of strange and charm quarks on these ensembles are set to their physical values, while the light sea quark masses are set such that $m_{s} / m_{l}=5$. The lattice spacings as measured using the $r_{1}$ parameter for the set of ensembles used here are 0.1207(11), 0.0888(8), and $0.0582(5) \mathrm{fm}$, respectively [61]. Further details of these lattice QCD ensembles can be found in Ref. [61].

In the valence sector, for light, strange, and charm quarks, we employ the overlap fermion action [63,64], which has exact chiral symmetry at finite lattice spacings [63-65] and is automatically $\mathcal{O}(m a)$ improved. The numerical implementation of the overlap fermion is carried out following the methods in Refs. [66,67]. A wall source smearing is utilized to calculate the light to charm quark overlap propagators on Coulomb gauge fixed lattices. In Table I, we list the quark masses and corresponding pion masses that we use for this calculation. The strange quark mass is tuned by equating the lattice estimate of the $\bar{s} s$ pseudoscalar meson mass to 688.5 MeV [68-70]. We follow the Fermilab prescription of heavy quarks for tuning the charm quark mass [71]. We tune it by equating the spin-averaged kinetic mass of the $1 S$ charmonia $\left[a \bar{M}_{\text {kin }}(1 S)=\frac{3}{4} a M_{\text {kin }}(J / \psi)+\frac{1}{4} a M_{\text {kin }}\left(\eta_{c}\right)\right]$ to

TABLE I. Parameters of ensembles used in this work.

\begin{tabular}{|c|c|c|c|}
\hline$N_{s}^{3} \times N_{t}$ & $\mathrm{a}(\mathrm{fm})$ & $a m_{q}$ & $m_{\pi}(\mathrm{MeV})$ \\
\hline $24^{3} \times 64$ & $0.1207(14)$ & $\begin{array}{l}0.0738 \\
0.054 \\
0.045 \\
0.038 \\
0.030 \\
0.024 \\
0.020 \\
0.0165 \\
0.0125 \\
0.0090 \\
0.0075 \\
0.0060 \\
0.0051 \\
0.0042 \\
0.0028\end{array}$ & $\begin{array}{l}689 \\
589 \\
539 \\
497 \\
449 \\
400 \\
367 \\
337 \\
297 \\
257 \\
237 \\
216 \\
202 \\
186 \\
153\end{array}$ \\
\hline $32^{3} \times 96$ & $0.0888(5)$ & $\begin{array}{l}0.049 \\
0.030 \\
0.020 \\
0.016 \\
0.0135 \\
0.012\end{array}$ & $\begin{array}{l}688 \\
537 \\
441 \\
396 \\
367 \\
345\end{array}$ \\
\hline $48^{3} \times 144$ & $0.0582(5)$ & $\begin{array}{l}0.028 \\
0.025 \\
0.020 \\
0.018\end{array}$ & $\begin{array}{l}685 \\
645 \\
576 \\
545\end{array}$ \\
\hline
\end{tabular}

its experimental value, $3068.6 \mathrm{MeV}$ [22]. The tuned bare charm quark masses are found to be $0.528,0.427$, and 0.290 on coarse to fine lattices, respectively, all of which satisfy $m_{c} a \ll 1$, ensuring reduced discretization artifacts in this calculation. Details on the charm quark mass tuning can be found in Refs. [69,70].

For the bottom quarks, we employ a nonrelativistic QCD (NRQCD) formulation [72]. In the NRQCD Hamiltonian, we include all the terms up to $1 / M_{0}^{2}$ as well as the leading term of the order of $1 / M_{0}^{3}$, where $M_{0}=a m_{b}$ is the bare mass of the bottom quarks in lattice units [73]. The bottom quark propagators are obtained by the usual time evolution of the NRQCD Hamiltonian, $H=H_{0}+\Delta H$, where the interaction term, $\Delta H$, is given by

$$
\begin{aligned}
\Delta H= & -c_{1} \frac{\left(\Delta^{(2)}\right)^{2}}{8\left(a m_{b}\right)^{3}}+c_{2} \frac{i}{8\left(a m_{b}\right)^{3}}(\nabla \cdot \tilde{E}-\tilde{E} \cdot \nabla) \\
& -c_{3} \frac{1}{8\left(m_{b}\right)^{2}} \sigma \cdot(\nabla \times \tilde{E}-\tilde{E} \times \nabla)-c_{4} \frac{1}{2 a m_{b}} \sigma \cdot \tilde{B} \\
& +c_{5} \frac{\left(\Delta^{(4)}\right.}{24 a m_{b}}-c_{6} \frac{\left(\Delta^{(2)}\right)^{2}}{16\left(a m_{b}\right)^{2}} .
\end{aligned}
$$

Here, $c_{1} \ldots c_{6}$ are the improvement coefficients, and for the fine lattice, we use their tree level values, while for the coarser two lattices, we employ their nonperturbative values as estimated by the HPQCD Collaboration [74] on the same set of lattices. To tune the bottom quark mass, we first calculate the kinetic mass of the spin average $1 S$ bottomonia,

$$
a M_{\mathrm{Kin}}=\frac{3}{4} a M_{\mathrm{Kin}}(\Upsilon)+\frac{1}{4} a M_{\mathrm{Kin}}\left(\eta_{b}\right),
$$

from the relativistic energy-momentum dispersion relation $a M_{\text {Kin }}=\left((a p)^{2}-(a \Delta E)^{2}\right) /(2 a \Delta E)$, and then equate it with its experimental value. Details on the bottom quark mass tuning is given in Ref. [75].

With this setup of light, strange, charm, and bottom quark propagators, we proceed to calculate the tetraquark correlators from the interpolating fields with various flavorspin combinations that we discuss in the next section.

\section{FOUR-QUARK INTERPOLATING OPERATORS}

In this section, we describe four-quark interpolating fields (operators) that we employ in this work. We construct these operators with two heavy and two light quarks and with the total spin $J=0$ and 1. As in Ref. [25], for both spins, we construct two types of operators, with the goal that one overlaps onto a tetraquark state of given quantum numbers and the other one overlaps onto the lowest strong decay two-meson states of the same quantum numbers. The tetraquark-type operators are constructed using the diquark prescription of Jaffe $[31,32]$ where a color neutral hadronic 
operator is constructed as a product of diquarks and antidiquarks. These diquarks (antidiquarks) can be in the $\overline{\mathbf{3}}_{c}\left(\mathbf{3}_{c}\right)$ or $\mathbf{6}_{c}\left(\overline{\mathbf{6}}_{c}\right)$ of the color SU(3) irreducible representation (irreps). Phenomenologically, the one gluon exchange model [31,32] favors an attractive interaction of two quarks and is in the $\overline{\mathbf{3}}$ irrep of $\mathrm{SU}(3)$. In this work, we construct tetraquark operators with both irreps of $\mathrm{SU}(3)$.

In the spin $J=1$ sector, we use diquarks and antidiquarks with the following configuration:

$$
\left(l_{1}, l_{2}\right) \rightarrow\left(\overline{\mathbf{3}}_{c}, 0, F_{A}\right), \quad(\bar{Q}, \bar{Q}) \rightarrow\left(\mathbf{3}_{c}, 1, F_{s}\right) .
$$

The light quark $\left(l_{1}, l_{2} ; l_{1} \neq l_{2}\right)$ combinations are constructed with color, spin, and flavor degrees of freedom (d.o.f.) as antisymmetric and are restricted within $\subset(u, d, s, c)$. The heavy quark combination $(\bar{Q}, \bar{Q})$ is constructed with color antisymmetric $\mathbf{3}_{c}$, forced by $\left(l_{1}, l_{2}\right)$ being in the $\overline{\mathbf{3}}_{c}$, and since flavor is manifestly symmetric, the spin is also symmetric. This combination is restricted to only heavy flavors $\subset(\bar{c}, \bar{b})$ with a further restriction of $Q \neq l_{1} \neq l_{2}$. With these diquarks and antidiquarks, a spin-1 tetraquark-type operator of flavor $\left(l_{1} l_{2} \bar{Q} \bar{Q}\right)$ is constructed as

$$
\mathcal{T}^{\mathbf{1}}(x)=\left(l_{1}\right)_{\alpha}^{a}(x)\left(C \gamma_{5}\right)_{\alpha \beta}\left(l_{2}\right)_{\beta}^{b}(x) \bar{Q}_{\kappa}^{a}(x)\left(C \gamma_{i}\right)_{\kappa \rho} \bar{Q}_{\rho}^{b}(x) .
$$

The label $x$ is a shorthand notation for $(\vec{x}, t)$, where $\vec{x}$ is the spatial local site and $t$ is the time slice. We then construct the two-meson-type operators corresponding to each flavor of the $\left(l_{1} l_{2} \bar{Q} \bar{Q}\right)$ tetraquark operator, $\mathcal{T}^{\mathbf{1}}(x)$, with the appropriate flavor antisymmetry as

$$
\begin{aligned}
\mathcal{M}^{1}(x) & =M_{1}(x) M_{2}^{*}(x)-M_{2}(x) M_{1}^{*}(x) \\
M_{1,2}(x) & =\left(l_{1,2}\right)_{\alpha}^{a}(x)\left(\gamma_{5}\right)_{\alpha \beta} \bar{Q}_{\beta}^{a}(x) \\
M_{1,2}^{*}(x) & =\left(l_{1,2}\right)_{\alpha}^{a}(x)\left(\gamma_{i}\right)_{\alpha \beta} \bar{Q}_{\beta}^{a}(x) .
\end{aligned}
$$

The tetraquark operator $\mathcal{T}^{\mathbf{1}}(x)$ is related to the two-meson product $M_{1}(x) M_{2}^{*}(x)$ via a Fierz transformation, and the relation is explicitly shown in the Appendix of Ref. [76] with the appropriate change in flavor labels. The various flavor and isospin $(I)$ combinations that we explore for these spin-1 tetraquark-type and two-meson-type operators are tabulated in Table II.

For the spin-0 sector, we employ following diquark/ antidiquark configuration in which both diquarks are with spin 0:

$$
(l, l) \rightarrow\left(\mathbf{6}_{c}, 0, F_{S}\right), \quad(\bar{Q} \bar{Q}) \rightarrow\left(\overline{\mathbf{6}}_{c}, 0, F_{s}\right) .
$$

The combination $(l, l)$ being manifestly flavor symmetric requires the color d.o.f. to be in the $\mathbf{6}_{c}$. For the combination $(\bar{Q}, \bar{Q})$, the color d.o.f. is consequently restricted to $\overline{\mathbf{6}}_{c}$, while the flavor d.o.f. is manifestly symmetric. In the above expression, for the combination $(l, l)$, we incorporate the
TABLE II. The tetraquark-type and two-meson-type operators that we study in this work with possible flavor combinations and allowed isospin $(I)$ in the spin-1 sector. The last column shows the range of pion masses that we use for the light quarks on the coarsest lattice spacing.

\begin{tabular}{lccc}
\hline \hline$\left(l_{1} l_{2} \bar{Q} \bar{Q}\right)$ & {$\left[\left(M_{1} M_{2}^{*}\right)\left(M_{2} M_{1}^{*}\right)\right]$} & $I$ & $m_{\pi}(\mathrm{MeV})$ \\
\hline$u d \bar{b} \bar{b}$ & $\left(B B^{0 *}\right)\left(B^{0} B^{*}\right)$ & 0 & $(257-688)$ \\
$u s \bar{b} \bar{b}$ & $\left(B B_{s}^{*}\right)\left(B_{s} B^{*}\right)$ & $\frac{1}{2}$ & $(186-688)$ \\
$u c \bar{b} \bar{b}$ & $\left(B B_{c}^{*}\right)\left(B_{c} B^{*}\right)$ & $\frac{1}{2}$ & $(153-688)$ \\
$u d \bar{c} \bar{c}$ & $\left(D D^{0 *}\right)\left(D^{0} D^{*}\right)$ & 0 & $(257-688)$ \\
$u s \bar{c} \bar{c}$ & $\left(D D_{s}^{*}\right)\left(D_{s} D^{*}\right)$ & $\frac{1}{2}$ & $(257-688)$ \\
\hline \hline
\end{tabular}

flavors $(u, s, c)$, while both $c$ and $b$ are used for $Q$. A spin-0 tetraquark-type operator of flavor $(l l \bar{Q} \bar{Q})$ constructed from the product of the aforementioned diquarks and antidiquarks is given by

$$
\mathcal{T}^{\mathbf{0}}(x)=l_{\alpha}^{a}(x)\left(C \gamma_{5}\right)_{\alpha \beta} l_{\beta}^{b}(x) \bar{Q}_{\kappa}^{b}(x)\left(C \gamma_{5}\right)_{\kappa \rho} \bar{Q}_{\rho}^{a}(x) .
$$

As previously done, we also construct a two-meson-type operator with the same quantum number as that of $(l l \bar{Q} \bar{Q})$, and it is given by

$$
\mathcal{M}^{0}(x)=\bar{Q}_{\alpha}^{a}(x)\left(\gamma_{5}\right)_{\alpha \beta} l_{\beta}^{a}(x) \bar{Q}_{\kappa}^{b}(x)\left(\gamma_{5}\right)_{\kappa \rho} l_{\rho}^{b}(x) .
$$

In Table III, we tabulate the spin-0 tetraquark configurations with the possible flavor combinations with the above flavor-spin configurations.

With the operators so constructed, we proceed to compute the correlation matrices of all the possible combinations of these operators for a given spin and flavor and then extract the associated energy states from the generalized eigenvalue solutions. In the next section, we discuss this in detail.

TABLE III. The tetraquark-type and two-meson-type operators for various flavors of in the spin- 0 sector. The range of pion masses used for $u u \bar{b} \bar{b}$ and $u u \bar{c} \bar{c}$ states is indicated in the last column. All other states are computed at their physical quark mass.

\begin{tabular}{lccc}
\hline \hline$\left(l_{1} l_{2} \bar{Q} \bar{Q}\right)$ & $\left(M_{1} M_{2}\right)$ & $I$ & $m_{\pi}(\mathrm{MeV})$ \\
\hline$u u \bar{b} \bar{b}$ & $(B B)$ & 1 & $(337-688)$ \\
$u u \bar{c} \bar{c}$ & $(D D)$ & 1 & $(297-688)$ \\
$s s \bar{b} \bar{b}$ & $\left(B_{s} B_{s}\right)$ & 0 & $\ldots$ \\
$c c \bar{b} \bar{b}$ & $\left(B_{c} B_{c}\right)$ & 0 & $\ldots$ \\
$s s \bar{c} \bar{c}$ & $\left(D_{s} D_{s}\right)$ & 0 & $\ldots$ \\
\hline \hline
\end{tabular}




\section{RESULTS}

In this section, first we elaborate the analysis procedure that we utilize to extract the energy levels from the matrix of correlation functions constructed from the interpolating fields mentioned above. Results obtained will be discussed after that.

\section{A. Analysis methods}

To evaluate the energy levels corresponding to the operators discussed in Sec. III, we first construct a correlator matrix of these operators and then use the variational method [40,41]. This matrix of correlation functions $C_{i j}(t)$ is given as

$$
C_{i j}(t)=\sum_{\vec{x}}\left\langle 0\left|\mathcal{O}_{i}(\vec{x}, t) \mathcal{O}_{j}^{\dagger}(\overrightarrow{0}, 0)\right| 0\right\rangle,
$$

where the operator $\mathcal{O}_{i}(\vec{x}, t) \in\left\{\mathcal{T}^{k}(\vec{x}, t), \mathcal{M}^{k}(\vec{x}, t)\right\}$ is either a tetraquark-type operator or a two-meson-type operator of a particular spin $k$. For the spin-1 tetraquark states, $\mathcal{O}_{i}$ 's correspond to Eqs. (4) and (5), whereas for the spin-0 states, these are from Eqs. (7) and (8). We analyze each spin sector separately. After constructing the correlation matrix, $\mathbf{C}(t)$, for a given spin and flavor combination, we solve a generalized eigenvalue problem (GEVP) to obtain the two energy levels [40,41]. The standard methods for GEVP $[40,41,77,78]$ are typically suited for a Hermitian correlator matrix. We note that, since we are using a wall source, the correlator matrix is non-Hermitian. ${ }^{3}$ Hence, we employ a variation of the GEVP method, named the eigenvector method, involving eigenvector projection in evaluating the ground state energies [79]. The method involves using the left and right eigenvectors of the correlator matrix to construct the principal correlator as discussed below:

(1) Compute left and right eigenvectors of the correlator matrix $\mathbf{C}(t)$ at chosen time slices $\left(t_{1}, t_{0}\right)$ as

$$
\begin{aligned}
& \mathbf{C}\left(t_{1}\right) v_{R, n}\left(t_{1}, t_{0}\right)=\lambda_{n}\left(t_{1}, t_{0}\right) \mathbf{C}\left(t_{0}\right) v_{R, n}\left(t_{1}, t_{0}\right) \\
& v_{L, n}\left(t_{1}, t_{0}\right) \mathbf{C}\left(t_{1}\right)=\lambda_{n}\left(t_{1}, t_{0}\right) v_{L, n}\left(t_{1}, t_{0}\right) \mathbf{C}\left(t_{0}\right) .
\end{aligned}
$$

The time slices $\left(t_{1}, t_{0}\right)$ are chosen such that $t_{1} / t_{0}>2$ and $t_{1}$ chosen in the region where the correlator is expected to be dominated by the ground state.

(2) The eigenvectors $v_{L, R, n}\left(t_{1}, t_{0}\right)$ are then used to construct the principal correlator as

$$
\Lambda_{n}(t)=v_{L, n}^{\dagger}\left(t, t_{0}\right) \mathbf{C}(t) v_{R, n}\left(t_{1}, t_{0}\right),
$$

and the effective masses are then obtained from $m_{n, \text { eff }}=\log \left(\Lambda_{n}(t) / \Lambda_{n}(t+\delta t)\right)$.

\footnotetext{
${ }^{3}$ The same correlator matrix is found to be Hermitian when computed with the unsmeared point sources and sink.
}

For a Hermitian correlator matrix, the left and right eigenvectors will be identical, and hence this method will be the same as standard methods [40,41,77,78]. For a non-Hermitian correlator, the source and sink operators are accordingly rotated by the left and right eigenvectors, respectively. To check the effects of non-Hermiticity, we also solve the GEVP with the standard methods $[40,41,77,78]$. We find that results obtained with either GEVP methods are consistent with each other, while the results from the eigenvector method is observed to be more stable.

The principal correlators thus obtained correspond to two energy levels, and the ground state energy is computed from the lowest one. On the other hand, we calculate the noninteracting two-meson threshold from the sum of the ground state masses of the two mesons involved. We then compare the lowest energy level obtained from the GEVP solution with the noninteracting two-meson threshold and evaluate the energy splitting between them as

$$
\Delta E^{k}=E_{\mathcal{T}^{k}}-E_{2 M},
$$

where $E_{\mathcal{T}^{k}}$ is the ground state energy obtained from the principal correlator of the GEVP, while $E_{2 M}=E_{M_{1}}+E_{M_{2}}$ is the energy of the noninteracting two-meson $\left(M_{1}\right.$ and $\left.M_{2}\right)$ threshold. The above energy splitting $\left(\Delta E^{k}\right)$ can be evaluated directly by fitting the two datasets separately and then computing the difference on each resample. Alternatively, this can also be evaluated by taking the jackknife ratio of the principal correlator $[\Lambda(t)]$ of the GEVP to two-meson correlators, $\left.M_{1}(t) \times M_{2}(t)\right)$, as

$$
\Lambda^{\prime}(t)=\frac{\Lambda(t)}{M_{1}(t) \times M_{2}(t)} \rightarrow \mathcal{A} e^{-\Delta E^{k} t}+\cdots
$$

A fit to the ratio correlator $\left[\Lambda^{\prime}(t)\right]$ will then directly yield the energy splitting with respect to the relevant threshold. Such a construction offers the advantage of reducing the systematic errors through jackknifing. However, in using such an effective correlator, caution must be exercised as this construction can produce spurious effects since the saturation of the ground states of the numerator and the denominator may not happen at the similar time slices. In this work, in estimating the energy splitting, we utilize both the direct and ratio methods and find consistent results. However, as expected, we find smaller uncertainties in the ratio method. We now present the results obtained through the above-mentioned analysis.

\section{B. Spin one tetraquarks $\boldsymbol{J}^{P}=\mathbf{1}^{+}$}

We begin with presenting data for the spin-1 doubly bottom tetraquark states. As described earlier, we compute a matrix of correlation functions of the tetraquark $\mathcal{T}^{\mathbf{1}}(x)$ and two-meson operators $\mathcal{M}^{\mathbf{1}}(x)$. The diagonal correlators of this matrix correspond to the same source-sink operators, 


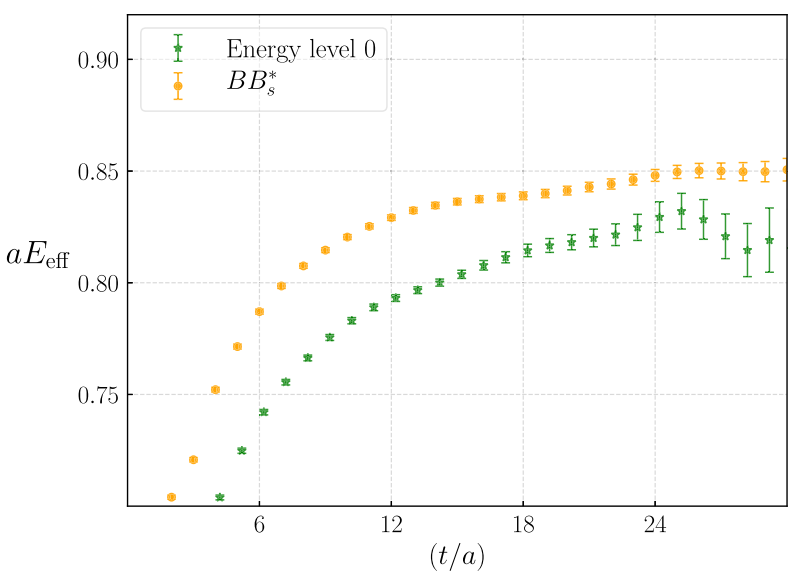

FIG. 1. Effective mass of the ground state energy level (data in green) obtained from the GEVP solution for the spin $1, u s \bar{b} \bar{b}$ tetraquark state at $m_{\pi}=688 \mathrm{MeV}$ and $a=0.0582 \mathrm{fm}$. The data in orange are the effective mass of the threshold correlator $B B_{s}^{*}$.

while the off-diagonal correlators have a tetraquark operator at the source and a two-meson operator at the sink and vice versa. The correlator matrix is non-Hermitian, and as mentioned earlier, in obtaining our final results, we employ the eigenvector method of diagonalization.

As a representative plot on analysis, in Fig. 1, we show the effective mass of the lowest energy level obtained from such a diagonalization along with the effective mass of the noninteracting two-meson threshold correlator for the case of $u s \bar{b} \bar{b}$. The data in orange are the effective mass of the noninteracting two-meson correlator, which in this case is obtained from the product of the correlators of the $B$ and $B_{s}^{*}$ mesons. ${ }^{4}$ The data in green are the effective mass of the lowest eigenvalue (the ground state), which is clearly below the effective mass of the threshold correlator. We also find that the effective mass corresponding to second eigenvalue overlaps with the effective mass of the threshold correlator in its approach to the plateau. However, as expected, it is noisier and needs a bigger basis of operators to extract it reliably. As discussed previously, for each flavor combination, we calculate the energy splitting $\Delta E^{\mathbf{1}}$ directly from Eq. (12) by fitting the individual correlators as well as from the ratio of correlators using Eq. (13).

Following the above procedure, we calculate the energy splittings $\left(\Delta E^{\mathbf{1}}\right)$ for all the doubly bottom tetraquarks with various flavor combinations mentioned in Table II. This is performed on three different lattices $(a \sim 0.12,0.09$, and $0.06 \mathrm{fm}$ ), and on each one, we vary the light quark masses over a wide range as listed in Table II. In Fig. 2, we show

\footnotetext{
${ }^{4}$ In the case of the $u s \bar{b} \bar{b}$ state, there exist two relevant threshold states, namely, $B B_{s}^{*}$ and $B_{s} B^{*}$. Of these two, we choose $B B_{s}^{*}$, which has relatively lower energy than that of $B_{s} B^{*}$. Similarly, for all other flavor combinations, such as $u c \bar{b} \bar{b}$, $u s \bar{c} \bar{c}$, and $s c \bar{b} \bar{b}$, we again choose the lowest strong decay threshold.
}

these results; in the left panel, we plot these energies computed at various pion masses. The results for the flavor combinations, $u q \bar{b} \bar{b}$ with $q \in(d, s, c)$, are shown by red, green, and blue colored data, respectively. For a representative plot, we choose to show results at the coarse lattice spacing since here we have the maximum number of pion masses and therefore can show the pion mass dependence of these energy splittings $\left(\Delta E^{\mathbf{1}}\right)$ more prominently. The result for the $u d \bar{b} \bar{b}$ state exhibits larger uncertainties at lower pion masses due to the presence of two light quarks, while the state $u s \bar{b} \bar{b}$ allows us to extract results at much lower pion masses. For $u c \bar{b} \bar{b}$, we could extract results even at the physical light quark mass.

It can be noted that for all the flavor combinations there is a trend of increment of $\Delta E^{1}$ with the lowering of pion masses, and we will discuss the details shortly. The availability of a large number of data points allows us to perform the chiral extrapolation very reliably. At each lattice spacing, we first perform the chiral extrapolation of $\Delta E^{1}$ and then perform a continuum extrapolation from the results obtained at three lattice spacings. We use the following simple quadratic Ansatz for both chiral and continuum extrapolations:

$$
\begin{aligned}
& \Delta E_{m_{\pi}}^{k}=c_{1}^{k}+c_{2}^{k} m_{\pi}^{2}, \\
& \Delta E_{a}^{k}=c_{1}^{k, a}+c_{2}^{k, a} a^{2} .
\end{aligned}
$$

Here, the label $k$ for the spin is kept general since we will also use these Ansätze for both spin sectors. We perform two fittings: one including all data points to show the pion mass dependence over a wide range of pion masses and the other with only the lower few pion masses to perform the chiral extrapolation. The fit results are shown in Table IV, in which in the second column we show the relevant slope parameter labeled as $c_{2}^{\mathbf{1}, m_{\pi}}$, which is indicative of the pion mass dependence of the energy splitting $\Delta E^{\mathbf{1}}$. It is instructive to compare $c_{2}^{1, m_{\pi}}$ parameters for different tetraquark states with different flavor combinations at a given lattice spacing. The fits indicate that the state $u d \bar{b} \bar{b}$ exhibits the most pronounced trend in the increase of $\Delta E^{1}$, followed by the state $u s \bar{b} \bar{b}$, while the state $u c \bar{b} \bar{b}$ exhibits a very minute variation. The results at the finest lattice spacings do not indicate such a clear trend as we do not have data points at much lighter pion masses at this lattice spacing.

For the second fit, i.e., for the chiral extrapolation, we use the Ansatz in Eq. (14) and employ cuts on the largest pion masses and include data corresponding to as low pion masses as can be afforded by meaningful uncertainties in the extrapolation. The results of the chiral extrapolation are shown in Table IV with the appropriate slope parameter labeled as $c_{2}^{\mathbf{1}, \text { chiral }}$ in column 5 , and the relevant maximum pion mass used in the fit being labeled as $m_{\pi}^{\text {cut }}$ is shown in column 4 . The chirally extrapolated values of $\left.\Delta E^{\mathbf{1}}\right|_{m_{\pi}^{\text {phy }}}$ are 

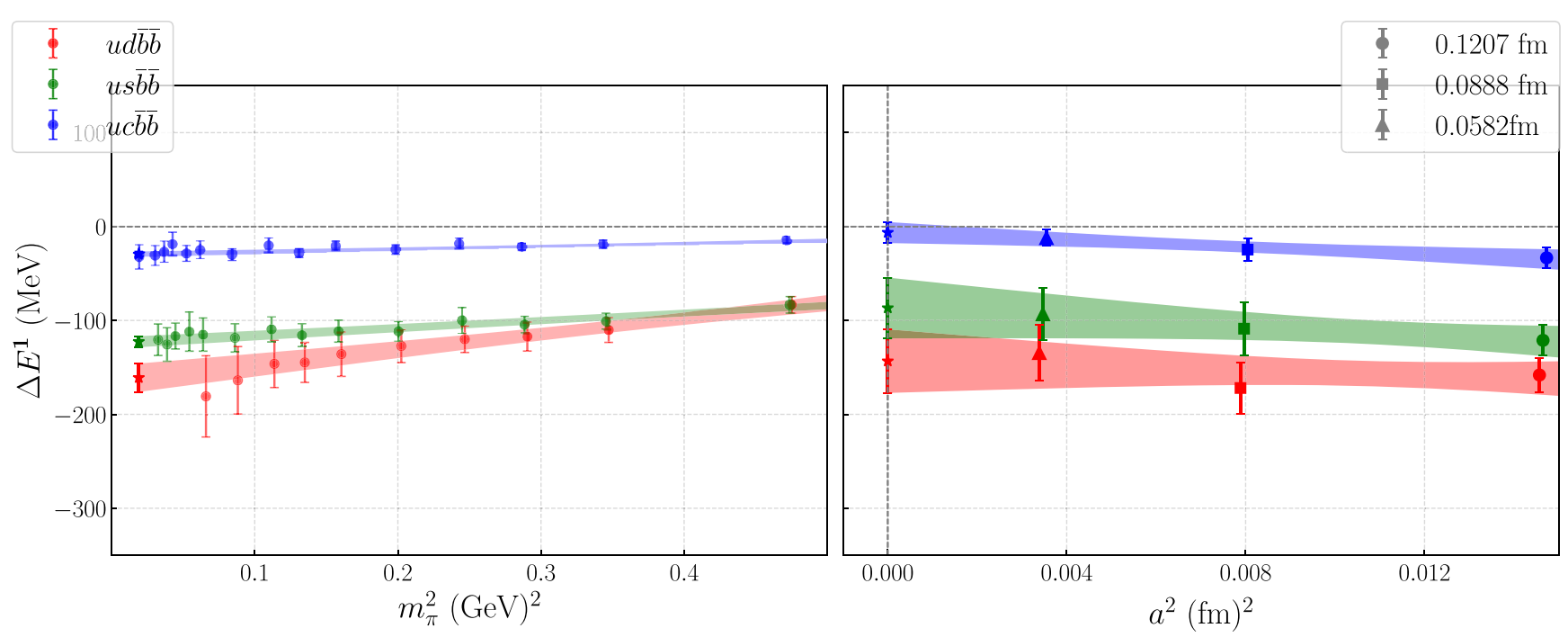

FIG. 2. Results of $u d \bar{b} \bar{b}, u s \bar{b} \bar{b}$, and $u c \bar{b} \bar{b}$ doubly bottom tetraquark states color coded in red, green, and blue, respectively, in both panels. Left panel: Energy splittings at several pion masses at $a=0.1207 \mathrm{fm}$ for each of the states. The fit bands indicate a chiral extrapolation fit as per Eq. (14) color coded appropriately for each state. Right panel: Continuum extrapolation results as per Eq. (15) from three lattice spacings. The data point at each lattice spacing is the result of the chiral extrapolation to the physical pion mass at that lattice spacing.

shown in the last column. We then use these chirally extrapolated $\left.\Delta E^{\mathbf{1}}\right|_{m_{\pi}^{\text {phys }}}$ from three different lattice spacings and perform a continuum extrapolation using the Ansatz in Eq. (15). The results of this extrapolation are shown in the right panel of Fig. 2, and the fit results are listed in Table V. The slope parameter $c_{2}^{\mathbf{1}, a}$ in this case will be an indicator of the lattice spacing dependence of the particular state. For $u d \bar{b} \bar{b}$ and $u s \bar{b} \bar{b}$, these are consistent with zero, indicating no dependence on lattice spacing. The parameter $c_{2}^{1, a}$ for

TABLE IV. Pion mass dependence and chiral extrapolation results for the spin-1 tetraquarks with different flavor combinations on three different lattices.

\begin{tabular}{cccccc}
\hline \hline State & $a(\mathrm{fm})$ & $c_{2}^{\mathbf{1 , \pi}}(\mathrm{MeV})$ & $m_{\pi}^{\text {cut }}$ & $c_{2}^{\mathbf{1} \text {,chiral }}$ & $\Delta E^{\left.\mathbf{1}\right|_{\pi} ^{\text {phys }}}(\mathrm{MeV})$ \\
\hline$u d \bar{b} \bar{b}$ & 0.1207 & $165(40)$ & 539 & $152(76)$ & $-158.1(18.0)$ \\
& 0.0888 & $246(71)$ & 688 & $246(71)$ & $-171.9(27.4)$ \\
& 0.0582 & $102(56)$ & 645 & $102(85)$ & $-134.3(29.6)$ \\
$u s \bar{b} \bar{b}$ & 0.1207 & $80(13)$ & 297 & $82(376)$ & $-121.2(16.4)$ \\
& 0.0888 & $91(55)$ & 537 & $130(133)$ & $-108.8(28.5)$ \\
& 0.0582 & $21(53)$ & 645 & $3(80)$ & $-93.1(27.8)$ \\
$u c \bar{b} \bar{b}$ & 0.1207 & $30(9)$ & 257 & $183(306)$ & $-33.3(10.9)$ \\
& 0.0888 & $21(14)$ & 441 & $71(89)$ & $-24.6(12.1)$ \\
& 0.0582 & $6(17)$ & 645 & $3(25)$ & $-12.0(8.6)$ \\
$u d \bar{c} \bar{c} \bar{c}$ & 0.1207 & $54(10)$ & 449 & $44(28)$ & $-31.4(5.8)$ \\
& 0.0888 & $43(17)$ & 688 & $43(17)$ & $-31.9(6.6)$ \\
& 0.0582 & $8(18)$ & 688 & $9(34)$ & $-18.5(11.9)$ \\
$u s \bar{c} \bar{c}$ & 0.1207 & $4(6)$ & 449 & $-8(9)$ & $-11.4(2.5)$ \\
& 0.0888 & $-7(11)$ & 537 & $-31(30)$ & $-10.2(3.8)$ \\
& 0.0582 & $-7(17)$ & 688 & $-7(17)$ & $-11.0(6.6)$ \\
\hline \hline
\end{tabular}

the $u c \bar{b} \bar{b}$ state indicates a mild dependence on the lattice spacing. The state $s c \bar{b} \bar{b}$, which is the $\mathrm{SU}(3)$ symmetric state of $u c \bar{b} \bar{b}$, requires no chiral extrapolation since all quark masses are at their physical values. The corresponding lattice spacing dependence parameter, $c_{2}^{\mathbf{1}, a}$, as shown in Table V, indicates no dependence on lattice spacing of this state. The continuum extrapolated results $\left.\Delta E^{\mathbf{1}}\right|_{a=0} ^{m_{a}^{\text {phys }}}$ are shown in Fig. 3.

It can be noted that at the finest lattice spacing the lowest pion mass available is $m_{\pi}=545 \mathrm{MeV}$, which may not be low enough for a chiral extrapolation. Because of this, the chirally extrapolated results at this lattice spacing may have a systematic effect arising from the absence of lower pion masses, and that may reflect in the lattice spacing dependence of some of our findings such as for the $u c \bar{b} \bar{b}$ state. Hence, we also report our results without including data

TABLE V. Continuum extrapolation results for the various flavors of tetraquark states in the spin-1 sector. The fourth column is the continuum extrapolation results from three lattices. The last column is obtained by averaging results from the coarser two lattices.

\begin{tabular}{lcccc}
\hline \hline State & $c_{1}^{\mathbf{1}, a}$ & $c_{2}^{\mathbf{1}, a}$ & $\left.\Delta E^{\mathbf{1}}\right|_{a=0} ^{m_{a}^{\text {phys }}}(\mathrm{MeV})$ & $\left.\Delta E^{\mathbf{1}}\right|_{\text {avg }}(\mathrm{MeV})$ \\
\hline$u d \bar{b} \bar{b}$ & $-143(34)$ & $-1239(2915)$ & $-143.3(33.9)$ & $-165.0(32.5)$ \\
$u s \bar{b} \bar{b}$ & $-87(32)$ & $-2393(2725)$ & $-86.7(32.4)$ & $-115.0(32.8)$ \\
$u c \bar{b} \bar{b}$ & $-6(11)$ & $-1918(1239)$ & $-6.4(11.2)$ & $-28.95(16.3)$ \\
$s c \bar{b} \bar{b}$ & $-8(3)$ & $-395(398)$ & $-7.67(3.21)$ & $-11.94(4.7)$ \\
$u d \bar{c} \bar{c}$ & $-23(11)$ & $-637(1001)$ & $-23.3(11.4)$ & $-31.7(8.8)$ \\
$u s \bar{c} \bar{c}$ & $-8(8)$ & $-241(574)$ & $-7.7(7.5)$ & $-10.8(4.5)$ \\
\hline \hline
\end{tabular}




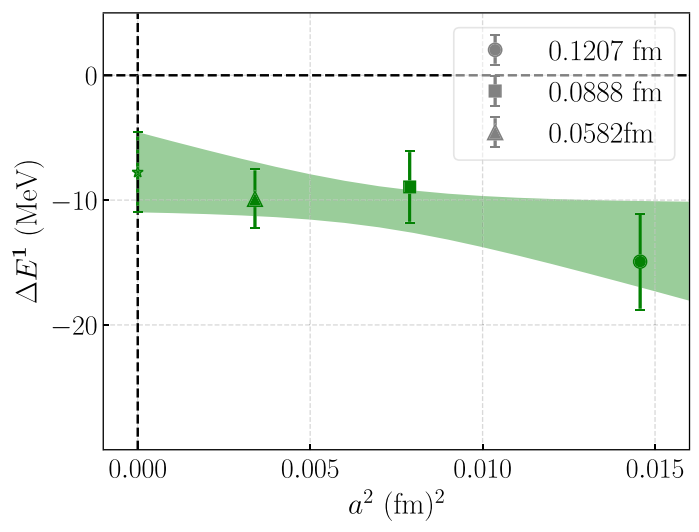

FIG. 3. Continuum extrapolation of the $s c \bar{b} \bar{b}$ state.

from the fine lattice. Since we are left with only two data points, we have not performed any fit (with 2 d.o.f.) in this case. Instead, we average the results obtained on those two lattices (with spacings 0.0888 and $0.1207 \mathrm{fm}$ ) and report that with error bars added in the quadrature. In column 5 of Table V, we show those average results by $\left.\Delta E^{1}\right|_{\text {avg. }}$.

We now discuss the results of the spin-1 doubly charm tetraquarks. In Fig. 4, we show those results where the left panel shows the pion mass dependence and the chiral extrapolation on the coarse lattice. The right panel represents results for the continuum extrapolation. The relevant lowest thresholds for the flavor combinations $u d \bar{c} \bar{c}$ and $u s \bar{c} \bar{c}$ are the noninteracting $D-D^{*}$ and $D-D_{s}^{*}$ mesons, respectively. For both cases, we find an energy level below their relevant strong decay thresholds, while the other energy level appears at the threshold. As in the doubly bottom cases, we calculate the energy splittings $\left[\Delta E^{1}\right.$ in Eq. (12)] between the lowest energy levels and the threshold states by direct fitting as well as from the ratio of correlators [as in Eq. (13)]. We represent the fitted results for $u d \bar{c} \bar{c}$ by red data points, while results for $u s \bar{c} \bar{c}$ are shown by green points. The fitted results for pion mass dependence and chiral extrapolation are shown in Table IV, while the results for continuum extrapolation are shown in Table V. In the case of $u d \bar{c} \bar{c}$, similar to $u d \bar{b} \bar{b}$, we observe a trend in the increase of $\Delta E^{1}$ with the lowering of the light quark constituents. This is evident from the fits for the pion mass dependence and is indicated by the $c^{\mathbf{1}, m_{\pi}}$ parameter on the coarsest two lattice spacings. The finest lattice spacing results do not clearly indicate this trend due to the lack of lower pion masses at that lattice spacing. The pion mass dependence of the energy splitting for $u s \bar{c} \bar{c}$, color coded in green, is much flatter in comparison to $u d \bar{c} \bar{c}$, and this trend is reflected in the $c_{2}^{\mathbf{1}, m_{\pi}}$ coefficient. The continuum extrapolations for both $u d \bar{c} \bar{c}$ and $u s \bar{c} \bar{c}$ indicate no discernible dependence on the lattice spacing.

In column 4 of Table $\mathrm{V}$, we show the continuum extrapolated results for doubly charmed tetraquarks. Column 5 shows the average results obtained the two coarser lattices. Both columns show the presence of energy levels below their respective thresholds for both $u d \bar{c} \bar{c}$ and $u s \bar{c} \bar{c}$. However, they are very close to their respective strong decay thresholds, as was also observed in Ref. [80]. Because of their close proximity to thresholds, a careful finite volume analysis [41] is needed to make conclusive statements about the nature of these states. Though they could be stable under strong interaction, they may not appear as bound states because of threshold effects.
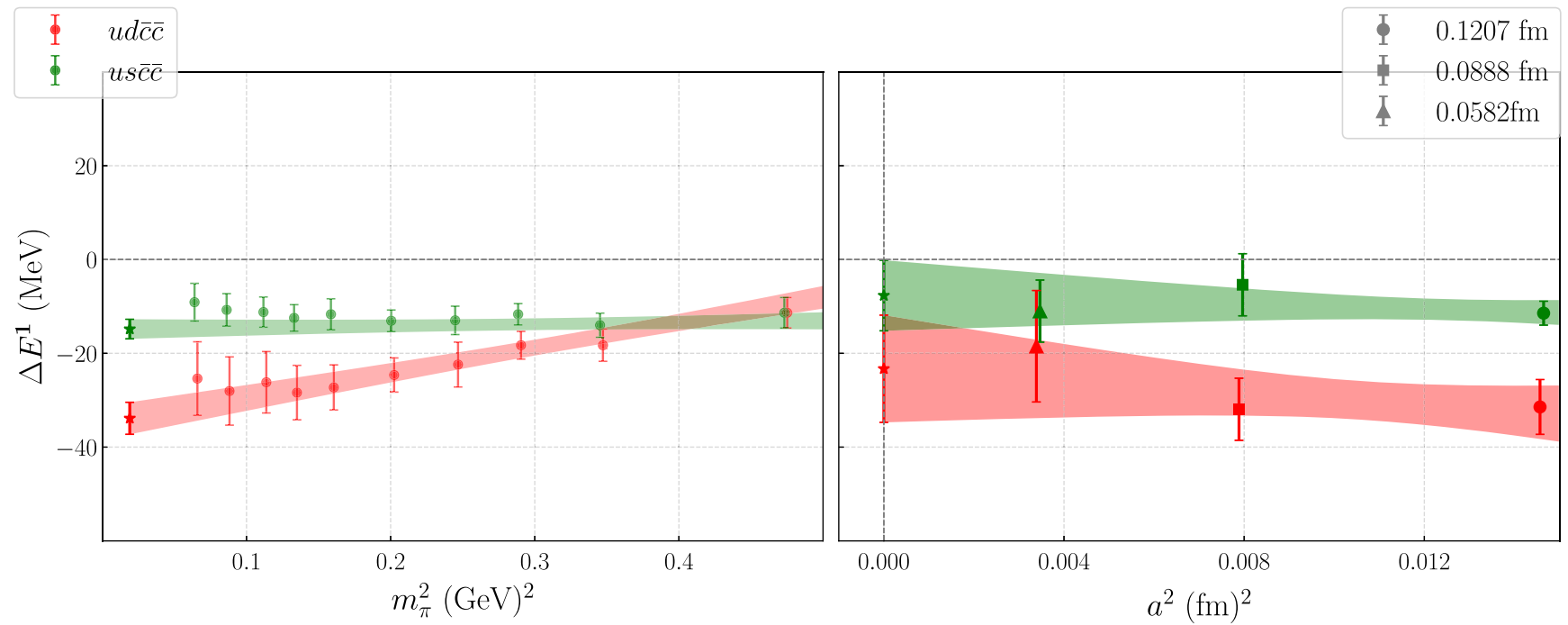

FIG. 4. Results of $u d \bar{c} \bar{c}$ and $u s \bar{c} \bar{c}$ doubly charm tetraquark states color coded in red and green in both panels. Left panel: Effective splittings at several pion masses at $a=0.1207 \mathrm{fm}$ for each of the states. The fit bands indicate a chiral extrapolation fit as per Eq. (14) color coded appropriately for each state. Right panel: Continuum extrapolation results as per Eq. (15) from three lattice spacings. The data point at each lattice spacing is the result of the chiral extrapolation to the physical pion mass at that lattice spacing. 


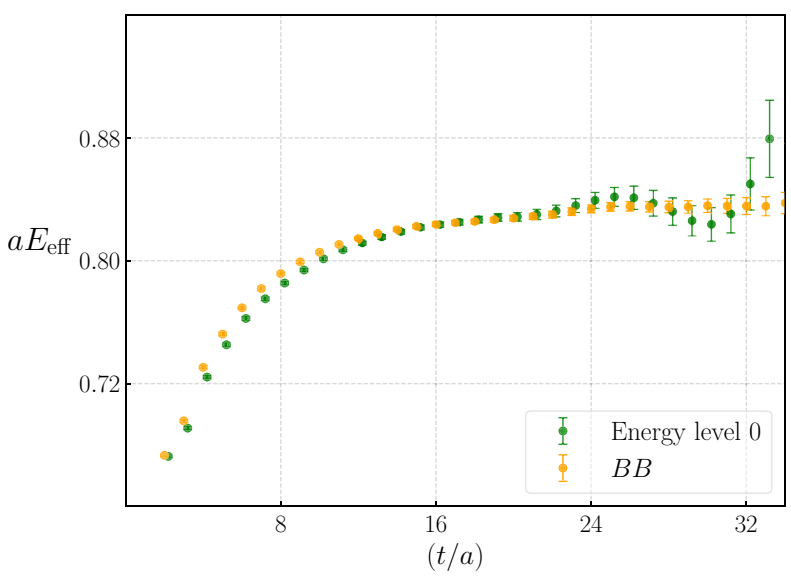

FIG. 5. Effective mass of the ground state energy level (green) obtained from the GEVP solution for the spin $0, u u \bar{b} \bar{b}$ tetraquark state. The data in orange is the effective mass of the threshold correlator $B B$. Results computed at $a=0.0583 \mathrm{fm}$ and at $m_{\pi}=688 \mathrm{MeV}$.

\section{Spin zero tetraquarks $J^{P}=0^{+}$}

In the spin-0 sector, we compute the energy levels of the tetraquark states with various flavor combinations that are listed in Table III. These tetraquark states are flavor symmetric cousins of those listed in Table II. As in the case of the spin-1 sector, we compute a matrix of correlation functions consisting tetraquark-type, $\mathcal{T}^{\mathbf{0}}(x)$, and two-meson-type, $\mathcal{M}^{0}(x)$, interpolating fields and employ the eigenvector method of diagonalization in obtaining our final results.

We shall begin by discussing the spin- 0 doubly charmed and doubly bottom tetraquark states with $I=1$. The effective masses of the principal correlators, obtained from GEVP analysis, for the flavor combination $u u \bar{b} \bar{b}$ are shown in Fig. 5. This representative figure is obtained on the fine lattice and at the pion mass $m_{\pi}=688 \mathrm{MeV}$. The relevant strong decay threshold in this case is the two noninteracting $B$ mesons. The effective mass of the product correlator of two $B$ mesons is represented by the orange data. The effective mass of the lowest eigenvalue, shown in green, is seen to coincide with the threshold correlator. This behavior is in contrast when compared with its flavor antisymmetric partner $u d \bar{b} \bar{b}$ where there is a clear indication of the ground state level being below the relevant threshold. The energy splitting $\left[\Delta E^{0}\right.$ in Eq. (12)] of the tetraquark state $u u \bar{b} \bar{b}$ is shown in the left panel of Fig. 6 by red colored data points, and results are obtained at various pion masses (on the coarser lattice) to explore the pion mass dependence. We note that the determination of these energy splittings is significantly noisier in comparison to the energy splittings in spin-1 $u d \bar{b} \bar{b}$ state with the same statistics. This limits us to using much lighter pion masses for $u u \bar{b} \bar{b}$. Furthermore, this also forces us to use the entire dataset for exploring both the pion mass dependence as well as the chiral extrapolation. We perform a chiral extrapolation with the Ansatz in Eq. (14) at each lattice spacing, and the results are listed in Table VI. The fits for the parameter $c_{2}^{\mathbf{0}, m_{\pi}}$ indicate a dependence on pion mass for $a=0.1207 \mathrm{fm}$, and no dependence is seen for the other two lattice spacings, since $c_{2}^{\mathbf{0}, m_{\pi}}$ is consistent with zero. It can be noted that this behavior again is in contrast with the pion mass dependence of the $u d \bar{b} \bar{b}$ state where a nontrivial dependence was clearly identified. After the chiral extrapolation, we perform the continuum extrapolation using the Ansatz in Eq. (15), and
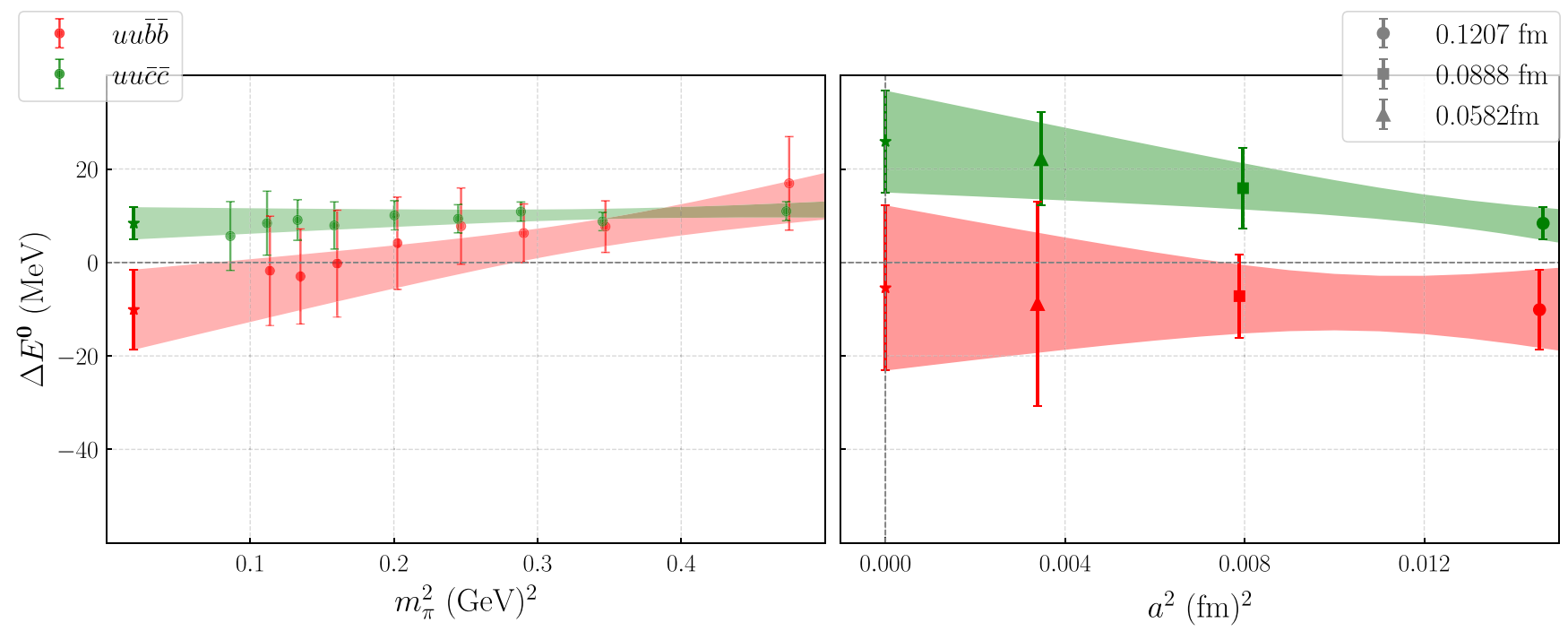

FIG. 6. Results of spin- $0 u u \bar{b} \bar{b}$ and $u u \bar{c} \bar{c}$ tetraquark states. Left panel: Energy splittings at several pion masses at $a=0.1207$ fm for both the states. The fit bands indicate a chiral extrapolation fit as per Eq. (14) color coded appropriately for each state. Right panel: Continuum extrapolation results as per Eq. (15) from three lattice spacings. The data point at each lattice spacing is the result of the chiral extrapolation to the physical pion mass at that lattice spacing. 
TABLE VI. Chiral continuum extrapolation results for various lattice spacings and flavors of tetraquark states in the spin- 0 sector.

\begin{tabular}{lcccc}
\hline \hline State & $a(\mathrm{fm})$ & $c_{1}^{\mathbf{0}, m_{\pi}}$ & $c_{2}^{\mathbf{0}, m_{\pi}}$ & $\left.\Delta E^{\mathbf{0}}\right|^{m_{\pi}^{\text {phys }}}(\mathrm{MeV})$ \\
\hline$u u \bar{b} \bar{b}$ & 0.1207 & $-11(9)$ & $50(25)$ & $-10.1(8.6)$ \\
& 0.0888 & $-8(9)$ & $26(26)$ & $-7.2(8.8)$ \\
& 0.0582 & $-9(23)$ & $33(57)$ & $-8.8(21.9)$ \\
$u u \bar{c} \bar{c}$ & 0.1207 & $8(4)$ & $6(10)$ & $8.4(3.4)$ \\
& 0.0888 & $16(9)$ & $-10(22)$ & $15.9(8.7)$ \\
& 0.0582 & $22(10)$ & $-12(24)$ & $22.2(10)$ \\
\hline \hline
\end{tabular}

fits are shown in Table VII. The slope parameter $c_{2}^{\mathbf{0}, a}$ for the state $u u \bar{b} \bar{b}$ is consistent with zero, indicating no dependence on the lattice spacing. The physical and continuum extrapolated result for $u u \bar{b} \bar{b}$ clearly indicates that there is no energy level below its lowest strong decay threshold with any statistical significance and is consistent with zero.

The green data points in Fig. 6 show the results for $\Delta E^{0}$ (on $a=0.1207 \mathrm{fm}$ lattice) for the spin- 0 doubly charmed tetraquarks $u u \bar{c} \bar{c}$. In this case, the GEVP solutions also display similar qualitative features, as the corresponding doubly bottom states where the ground state coincides with the threshold and a well-separated second state lies above that. Here, the threshold is that of the two noninteracting $D$ mesons. As in the previous case, we use the entire dataset for the pion mass dependence as well as chiral extrapolation. The chiral extrapolation fits at each lattice spacing shown in Table VI indicate no dependence on the pion mass since the parameter $c_{2}^{\mathbf{0}, m_{\pi}}$ is found to be consistent with zero. The continuum extrapolation for this case, color coded in green, is shown in the right panel of Fig. 6, which indicates a mild dependence on the lattice spacing. The physical and continuum extrapolated results $\left(\left.\Delta E^{1}\right|_{a=0} ^{m_{a}^{\text {phys }}}\right)$ are shown in the fifth column of Table VII, and all are found to lie above the respective threshold states. As in the spin-1 case, we have also calculated the average values of these energy splittings from the results obtained on two coarse lattices and show that in the last column of Table VII.

TABLE VII. Continuum extrapolation results for the various flavors of tetraquark states in the spin- 0 sector. The fourth column is the continuum extrapolation results from three lattices. The last column is obtained by averaging results from the coarser two lattices.

\begin{tabular}{ccccc}
\hline \hline State & $c_{1}^{\mathbf{0}, a}$ & $c_{2}^{\mathbf{0}, a}$ & $\left.\Delta E^{\mathbf{0}}\right|_{a=0} ^{m_{n}^{\text {phys }}}(\mathrm{MeV})$ & $\left.\Delta E^{\mathbf{0}}\right|_{\text {avg }}(\mathrm{MeV})$ \\
\hline$u u \bar{b} \bar{b}$ & $-5(18)$ & $-303(1549)$ & $-5.5(17.7)$ & $-8.7(12.3)$ \\
$u u \bar{c} \bar{c}$ & $26(11)$ & $-1202(824)$ & $25.9(10.9)$ & $12.15(9.3)$ \\
$s s \bar{b} \bar{b}$ & $3(9)$ & $328(1108)$ & $2.5(9.2)$ & $6.6(11)$ \\
$s s \bar{c} \bar{c}$ & $14(4)$ & $-319(356)$ & $14.1(3.9)$ & $11.1(4.1)$ \\
$c c \bar{b} \bar{b}$ & $16(1)$ & $-285(139)$ & $15.7(1.0)$ & $12.5(1.69)$ \\
\hline \hline
\end{tabular}

With our available quark propagators, we are also able to study $I=0, J=0$ tetraquark states, $s s \bar{b} \bar{b}, s s \bar{c} \bar{c}$, and $c c \bar{b} \bar{b}$, where the strange, charm, and bottom quark masses are tuned to their physical values. Energy levels obtained for these states will thus be at the physical points, and there is no need for any chiral extrapolation. The thresholds for these states are the noninteracting $B_{s} B_{s}, D_{s} D_{s}$, and $B_{c} B_{c}$, respectively. These require only a continuum extrapolation, which is shown in the two panels of Fig. 7, and the fitted results are shown in Table VII. The estimates of the energy splitting $\Delta E^{0}$ for the state $s s \bar{b} \bar{b}$ (color coded in red) show no lattice spacing dependence, and the final result is consistent with zero, indicating the absence of any bound state. For the state $s s \bar{c} \bar{c}$, we also find similar results, and the continuum extrapolated result lies above its respective threshold, which is most likely to be a scattering state. Results for the state $c c \bar{b} \bar{b}$ indicate a mild lattice spacing dependence, and the continuum result is also most likely be a scattering state. In conclusion, our analysis on the $I=0$, spin-0, tetraquarks with flavor combinations $s s \bar{b} \bar{b}, s s \bar{c} \bar{c}$, and $c c \bar{b} \bar{b}$ suggest the absence of any bound state, and the observed energy levels correspond to the scattering states. Recently, a potential based lattice QCD study in Ref. [59] for doubly bottom spin-0 states also concluded the same.

\section{Finite volume effects}

For all the spin-1 tetraquark states with various flavor combinations listed in Table II, we have found the energy levels below their respective strong decay thresholds. In some cases, the energy splittings $\left(\Delta E^{\mathbf{1}}\right)$ between the ground state and the threshold state are very large, while for others, they are close to and below their respective thresholds. However, all these energy levels are obtained within a single volume of about $3 \mathrm{fm}$. It is thus necessary to estimate the finite volume effects on these energy differences and obtain their infinite volume estimates, which can then be interpreted as the binding energies of the corresponding bound states. However, repeating these calculations on multiple lattice volumes is computationally very expensive and so is beyond the scope of this work.

However, it is possible to identify a few states for which the finite volume corrections will be suppressed, i.e., could be very small. The estimation of $\Delta E^{\mathbf{1}}$ on a single large enough volume for such a case, in fact, would be close to its binding energy $\left(B_{\infty}\right)$. As demonstrated in Refs. [81-83], the finite volume corrections $\Delta_{\mathrm{FV}}$ to energy levels corresponding to an infinite volume bound state with energy $E_{\infty}$ scale as

$$
\begin{aligned}
\Delta_{\mathrm{FV}} & =E_{\mathrm{FV}}-E_{\infty} \propto \mathcal{O}\left(e^{-k_{\infty} L}\right) / L, \\
\text { with } \quad k_{\infty} & =\sqrt{\left(m_{1}+m_{2}\right) B_{\infty}},
\end{aligned}
$$

where $E_{\mathrm{FV}}$ is the energy level computed a cubic lattice, $k_{\infty}$ is the binding momentum of the infinite volume state, and 


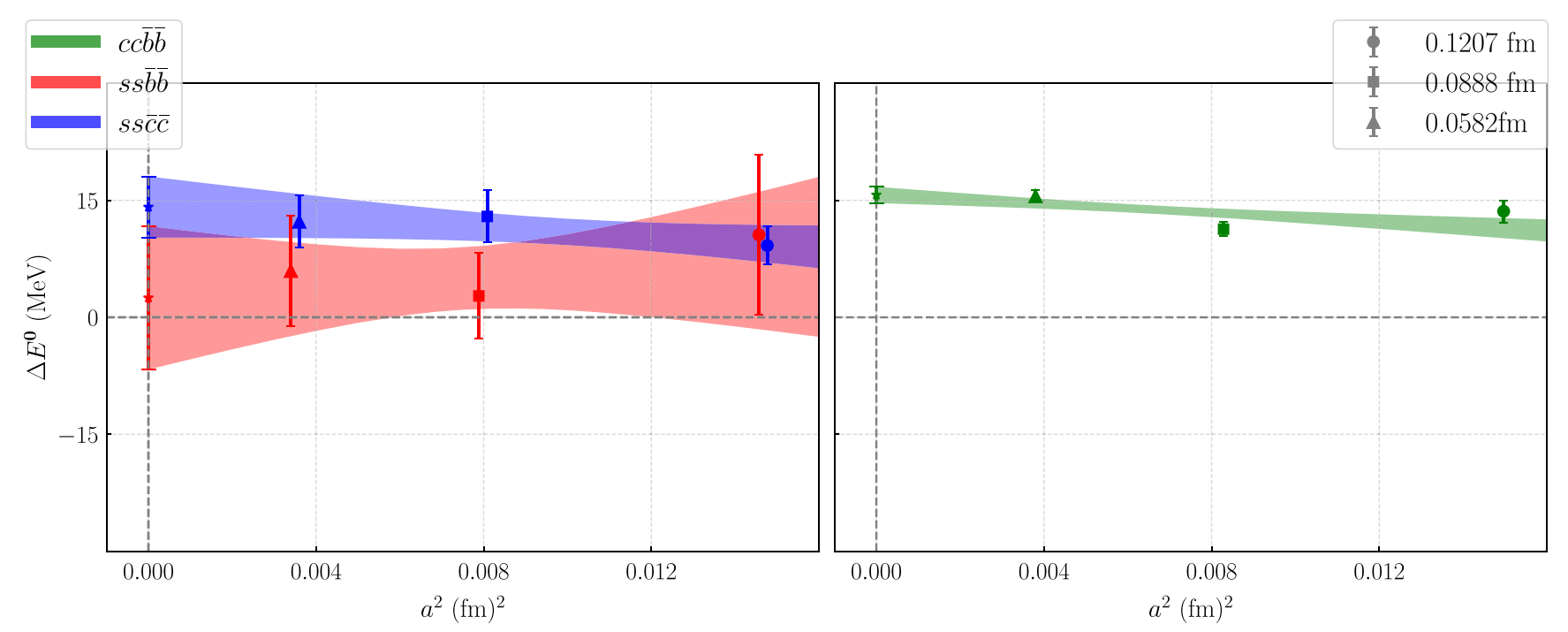

FIG. 7. Left: Continuum extrapolation of $s s \bar{b} \bar{b}$ and $s s \bar{c} \bar{c}$ states from three lattice spacings. Right: Continuum extrapolation of the $c c \bar{b} \bar{b}$.

$\left(m_{1}, m_{2}\right)$ are the masses of the two noninteracting particles with the threshold energy $m_{1}+m_{2}$. It should be noted from the above expression that the finite volume effects are suppressed by the threshold mass $\left(m_{1}+m_{2}\right)$ and that this suppression is significantly enhanced for the cases in which the threshold states are heavy mesons, such as those we are studying here. In addition to that, if $\Delta E$ is also large, then the finite volume corrections will further be suppressed since it also enters in the exponential. Therefore, in the doubly bottom sector, tetraquark states with the flavor combinations, $u d \bar{b} \bar{b}$ and $u s \bar{b} \bar{b}$, for which the $\Delta E$ values are found to be more than 150 and $100 \mathrm{MeV}$, respectively, will have small finite volume corrections. For these cases, it is quite natural to expect that the energy splitting $\Delta E$ will be closer to their infinite volume binding energy. Therefore, these states will be stable under strong interactions. However, for the cases, particularly for the doubly charmed tetraquarks, which are below but closer to their thresholds (i.e., $\Delta E$ values are closer to zero), it will be difficult to get any qualitative estimate for their finite volume corrections. In those cases, one needs to perform a detail finite volume study [41] to make any conclusive statement about their infinite volume pole structures.

\section{E. Heavy quark effective theory and hadron mass relations}

The Heavy quark effective theory (HQET) is a very useful tool and is often utilized to understand various properties of heavy hadrons including their energy spectra. Using heavy quark symmetries, one can also obtain mass relations between heavy flavored hadrons such as those mentioned in Ref. [24]. Using such symmetry relations, Ref. [24] predicted masses and binding energies of various tetraquarks states including some of those studied in this work. Although such relations are valid in the infinite quark mass limit, they are used at the bottom and even at the charm quark masses. It will therefore be interesting to investigate these relations by a first principles nonperturbative method, such as lattice QCD, with a goal to validate these relations at a given quark mass and access their deviation, if any, from the heavy quark limit. The availability of data on the ground state masses on mesons, baryons, and tetraquarks obtained from this calculation, both at the charm and the bottom quark masses, provides such an opportunity to systematically investigate these relations. Below, we elaborate that.

The work in Ref. [24] states the following relation among the hadrons with heavy quarks,

$$
\begin{gathered}
m\left(\left\{Q_{i} Q_{j}\right\}\left[\bar{q}_{k} \bar{q}_{l}\right]\right)-m\left(\left\{Q_{i} Q_{j}\right\} q_{y}\right) \\
=m\left(Q_{x}\left[q_{k} q_{l}\right]\right)-m\left(Q_{x} \bar{q}_{y}\right),
\end{gathered}
$$

where $Q_{i}$ and $q_{k}$ denote heavy and light quarks, respectively. Here, we use the same notation as in Ref. [24]. The braces $\{\ldots\}$ and $[\ldots]$ imply the symmetrization and antisymmetrization, respectively, with respect to the flavor d.o.f. In this notation, $\left(\left\{Q_{i} Q_{j}\right\}\left[\bar{q}_{k} \bar{q}_{l}\right]\right)^{5}$ represents a tetraquark operator with the flavor symmetries indicated by the braces, while $\left(\left\{Q_{i} Q_{j}\right\} q_{y}\right),\left(Q_{x}\left[q_{k} q_{l}\right]\right)$ and $\left(Q_{x} \bar{q}_{y}\right)$ represent a heavy-heavy-light baryon, heavy-light-light baryon, and heavy-light meson, respectively. It should be noted that Ref. [24] provides four such relations depending on the combination of flavor symmetrization/antisymmetrization, and the one shown here corresponds to our operator construction. The relation in Eq. (17) can then be employed

\footnotetext{
${ }^{5}$ The tetraquark operator used in this work is a complex conjugate of this operator.
} 

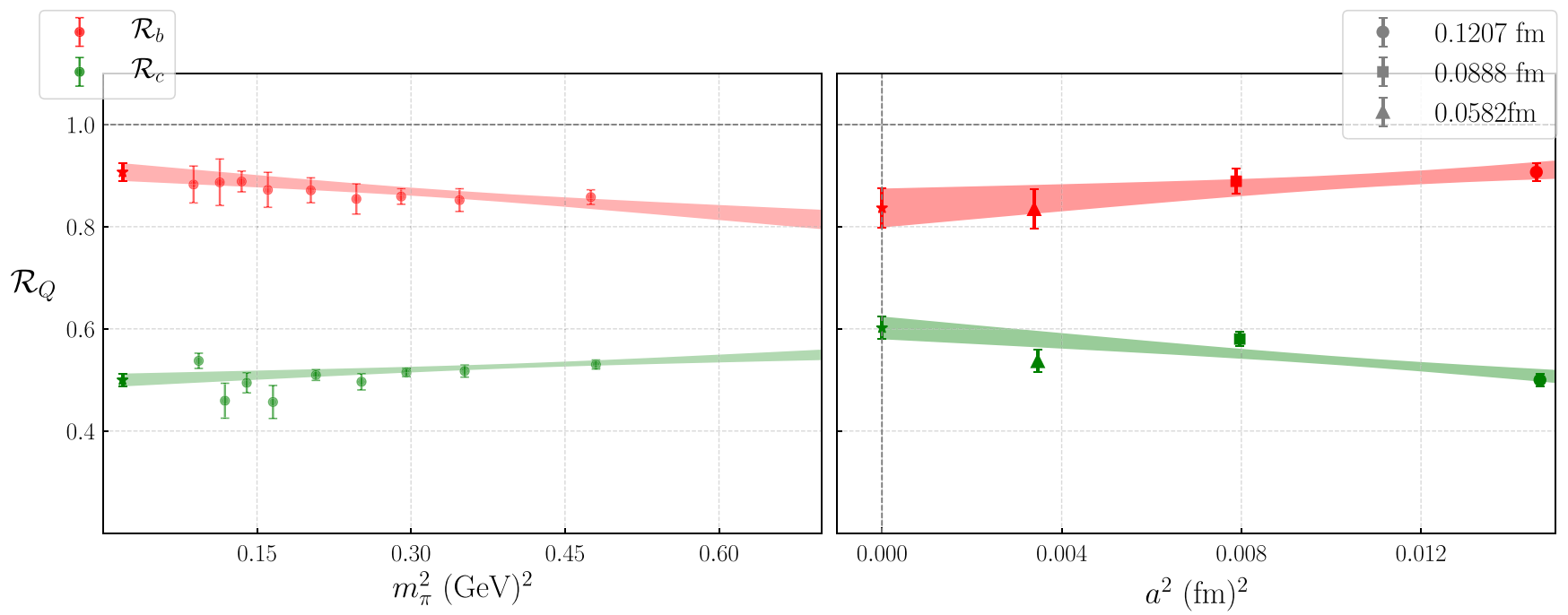

FIG. 8. Results of the ratios $\mathcal{R}_{b}$ and $\mathcal{R}_{c}$ color coded as red and green, respectively. Left: Results of the chiral extrapolation at $a=0.1207 \mathrm{fm}$. Right: Continuum extrapolation results from three lattice spacings.

to predict the masses of the tetraquark states by substituting the relevant masses of heavy baryons and mesons. In Ref. [24], this was calculated by using the spin average masses of the charmonia, bottomonia, and heavy baryons by inserting their experimental or quark model values.

Here, we aim to study this relation at both the charm and the bottom quark masses. We do not consider the spinaverage mass and instead use the spin- $1 / 2$ states for baryons and pseudoscalar mass for the heavy-light meson. Any deviation from the equality in Eq. (17) would be maximum in this choice. In doing so, we will be able to estimate an upper bound of the deviation from the heavy quark limit which originates from all $\left(1 / m_{Q}\right)^{n}$ corrections. In evaluating Eq. (17), we find it to be convenient ${ }^{6}$ to redefine the relation as a ratio which for the charm and bottom quarks is given by

$$
\mathcal{R}_{b} \equiv \frac{M_{u d \bar{b} \bar{b}}-M_{\Xi_{b b}}}{M_{\Lambda_{b}}-M_{B}}, \quad \mathcal{R}_{c} \equiv \frac{M_{u d \bar{c} \bar{c}}-M_{\Xi_{c c}}}{M_{\Lambda_{c}}-M_{D}}
$$

In the limit of infinitely heavy quarks, the ratio $\mathcal{R}_{Q}$ will be unity. In computing these ratios $\left(\mathcal{R}_{c / b}\right)$, we first evaluate the jackknife ratios of the following correlators,

$$
\begin{aligned}
& \frac{C_{u d \bar{b} \bar{b}}(t)}{C_{\Xi_{b b}}(t)} \rightarrow A^{\prime} e^{-\left(M_{u d \bar{b} \bar{b}}-M_{\Xi_{b b}}\right) t}+\cdots, \\
& \frac{C_{\Lambda_{Q}}(t)}{C_{M_{Q \bar{q}}}(t)} \rightarrow B^{\prime} e^{-\left(M_{\Lambda_{Q}}-M_{Q \bar{q}}\right) t}+\cdots,
\end{aligned}
$$

which directly provide the difference of masses as shown above. $\mathcal{R}_{c / b}$ are then evaluated from the fits to these ratio

\footnotetext{
${ }^{6}$ The use of the ratio of masses allows for the cancellation of lattice artifacts in addition to the cancellation of uncertainties from resampling.
}

correlators. In addition, we also fit the individual masses of tetraquarks, mesons, and baryons and calculate $\mathcal{R}_{c / b}$ from Eq. (18). We find consistent results with both methods, and the evaluation with Eq. (18) provides improved uncertainties. As we have access to a large number of light quark masses, while keeping the heavy quark mass at the charm and bottom quark, we vary the light quark mass and calculate $\mathcal{R}_{c / b}$ for each case. In Fig. 8, we show these results at several pion masses for the coarser lattice $(a \sim 0.12 \mathrm{fm})$ using the entire dataset in fitting. This is done for other lattice spacings as well. The results clearly indicate a wide separation of ratios between the charm and bottom quarks; while $\mathcal{R}_{b}$ is closer to the heavy quark limit of unity, $\mathcal{R}_{c}$ deviates from it substantially. After repeating this calculation on the other two lattices, we perform a simplistic chiral and continuum extrapolation according to the Ansatzes in Eqs. (14) and (15). The fit results are shown in Tables VIII and IX at three lattice spacings. For both ratios, $\mathcal{R}_{b}$ and $\mathcal{R}_{c}$, we do not observe any appreciable dependence on the pion mass as indicated by the parameter $c_{2}^{\pi}$ in Table VIII. In addition, the continuum extrapolation fit in Table IX does not indicate any lattice spacing

TABLE VIII. Chiral extrapolation of ratios $\mathcal{R}_{Q}$ for charm and bottom quarks.

\begin{tabular}{lllll}
\hline \hline Ratio & \multicolumn{1}{c}{$a$} & $c_{1}^{\pi}$ & $c_{2}^{\pi}$ & $\left.\mathcal{R}_{Q}\right|^{m_{\pi}^{\text {phys }}}$ \\
\hline $\mathcal{R}_{b}$ & 0.1207 & $0.91(2)$ & $-0.14(5)$ & $0.907(17)$ \\
& 0.088 & $0.89(3)$ & $-0.03(0.1)$ & $0.889(24)$ \\
& 0.058 & $0.83(4)$ & $0.05(0.1)$ & $0.835(38)$ \\
$\mathcal{R}_{c}$ & 0.1207 & $0.50(1)$ & $0.07(3)$ & $0.500(13)$ \\
& 0.088 & $0.58(1)$ & $-0.05(5)$ & $0.580(14)$ \\
& 0.058 & $0.54(2)$ & $0.03(6)$ & $0.537(22)$ \\
\hline \hline
\end{tabular}


TABLE IX. Continuum extrapolation of ratios $\mathcal{R}_{Q}$ for charm and bottom quarks.

\begin{tabular}{lcrc}
\hline \hline Ratio & $c_{1}^{a}$ & $c_{2}^{a}$ & $\left.\mathcal{R}_{Q}\right|_{a=0} ^{m_{a}^{\text {phys }}}$ \\
\hline $\mathcal{R}_{b}$ & $0.84(4)$ & $5.01(3.18)$ & $0.837(38)$ \\
$\mathcal{R}_{c}$ & $0.60(2)$ & $-6.33(2.01)$ & $0.602(22)$ \\
\hline \hline
\end{tabular}

dependence for the bottom and charm quarks. The continuum extrapolated results are listed in the last column of Table VIII; we find $\mathcal{R}_{b}=0.837(38)$ and $\mathcal{R}_{c}=0.602(22)$. These results clearly indicate that there is a substantial deviation from the heavy quark limit at the charm quark mass, implying there might be large contributions from $\left(1 / m_{Q}\right)^{n}$ corrections. However, results at the bottom quark mass are much closer to the heavy quark limit. Our results indicate that, as far as the heavy quark symmetry relations such as that is shown in Eq. (17) are considered, the charm quark mass is not heavy enough for the equality, and one certainly needs to incorporate appropriate leading order $1 / m_{Q}$ and then higher order correction terms. However, one can of course use these relations for bottom quarks with higher order $1 / m_{Q}$ corrections.

\section{DISCUSSION AND CONCLUSIONS}

Recently, there has been tremendous amount of activities in studying multiquark states both theoretically and experimentally. In particular, heavy tetraquarks are being investigated at various laboratories as well as studied theoretically through different models and by lattice QCD calculations. In this work, using lattice QCD, we have performed a detailed study on the doubly heavy tetraquark states with quark contents $q_{1} q_{2} \bar{Q} \bar{Q}, q_{1}, q_{2} \subset u, d, s, c$, and $Q \equiv b, c$, in both spin-0 $(J=0)$ and spin-1 $(J=1)$ sectors. Not only do we study $u d \bar{b} \bar{b}$ and $u s \bar{b} \bar{b}$, as was studied in Ref. [25], but we also explore $u c \bar{b} \bar{b}, u d \bar{c} \bar{c}$, and $u s \bar{c} \bar{c}$ states and additionally include the spin-0 sector of doubly heavy tetraquarks. In doing so, we have presented a systematic dependence of the ground state spectra of such states on their light quark constituents over a wide range of quark masses starting from the quark mass corresponding to the physical pion mass to the strange quark mass. Since all these hadrons involve heavy quarks, naturally, like any heavy flavored hadrons, they are susceptible to heavy quark discretization effects in a lattice calculation. To check the lattice spacing dependence, we have obtained results at three lattice spacings, the finest one being at $0.0582 \mathrm{fm}$. At a given lattice spacing, we perform a chiral extrapolation using several quark masses and then perform a continuum extrapolation to get the final results. For all the states in the spin-1 sector, we observe the presence of energy levels below their respective two-meson thresholds, the deepest one being for the doubly bottom tetraquark, $u d \bar{b} \bar{b}$. Furthermore, for various flavor combinations of the tetraquark states, we find that there is a clear trend of increase in the energy splitting $(\Delta E)$ as the light quark masses of such states are decreased and it becomes maximum at the physical quark mass. This energy splitting in the infinite volume limit of such a state can be interpreted as its binding energy. This trend was first indicated in the lattice calculation in Ref. [25] for the states $u d \bar{b} \bar{b}$ and $u s \bar{b} \bar{b}$. Here, we confirm that over a wide range of quark masses. Additionally, we find that such a trend holds for all the spin-1 states considered here, including the doubly charm tetraquark states. For the doubly charmed tetraquark states, $u d \bar{c} \bar{c}$ and $u s \bar{c} \bar{c}$, we also find that the ground states are below their respective thresholds. However, they are quite close to their thresholds, which was also observed in Ref. [80]. Though they could be stable under strong interactions, one needs to carry out finite volume analysis to establish their bound state properties, if there are any. We would also like to point out that most of these states, except $u c \bar{b} \bar{b}$, show either no discernible dependence or very mild dependence on lattice spacing. However, this will be clear when in a future study we include much lower pion masses on the fine lattice. Our final results for doubly heavy spin-1 tetraquarks states from this calculation are summarized in Table X. Our estimates for the $u d \bar{b} \bar{b}$ and $u s \bar{b} \bar{b}$ are in agreement with those of Ref. [25] at a lattice spacing (approximately $0.09 \mathrm{fm}$ ) where both of our data points are available.

We also provide a comparison of global results of spin-1 doubly heavy tetraquark states with various flavors and show that in Fig. 9. The results from Refs. [23,24] are based on HQET and the potential model, respectively, while the rest are lattice calculations. All results agree with the existence of deeply bound spin-1 tetraquark states, $u d \bar{b} \bar{b}$ and $u s \bar{b} \bar{b}$, which are stable under strong interactions. Our results for the doubly bottom states agree well with those from the HQET predictions [24] as well as that of the result in Ref [25] at similar lattice spacings (approximately $0.09 \mathrm{fm}$ ). Reference [25] used $N_{f}=2+1$ PACS-CS gauge field configurations and Coulomb gauge fixed wall sources with clover action in the valence sector. The results were extracted at a single lattice spacing $(a \sim 0.09 \mathrm{fm})$ at three pion masses, and a chiral extrapolation with $m_{\pi}^{2}$ was performed to obtain the final result. The results from Ref. [60] were obtained from the potential based lattice QCD study in which potentials of two $B$ mesons were computed in the static approximation for various spinisospin combinations. These were then fitted to a phenomenologically motivated Ansatz and were further used to solve a Schrödinger equation to determine a bound state.

TABLE $X$. Final results for the spin-1 tetraquarks.

\begin{tabular}{lccc}
\hline \hline State & $\Delta E^{\mathbf{1}}(\mathrm{MeV})$ & State & $\Delta E^{\mathbf{1}(\mathrm{MeV})}$ \\
\hline$u d \bar{b} \bar{b}$ & $-143(34)$ & $u s \bar{b} \bar{b}$ & $-87(32)$ \\
$u c \bar{b} \bar{b}$ & $-6(11)$ & $s c \bar{b} \bar{b}$ & $-8(3)$ \\
$u d \bar{c} \bar{c}$ & $-23(11)$ & $u s \bar{c} \bar{c}$ & $-8(8)$ \\
\hline \hline
\end{tabular}




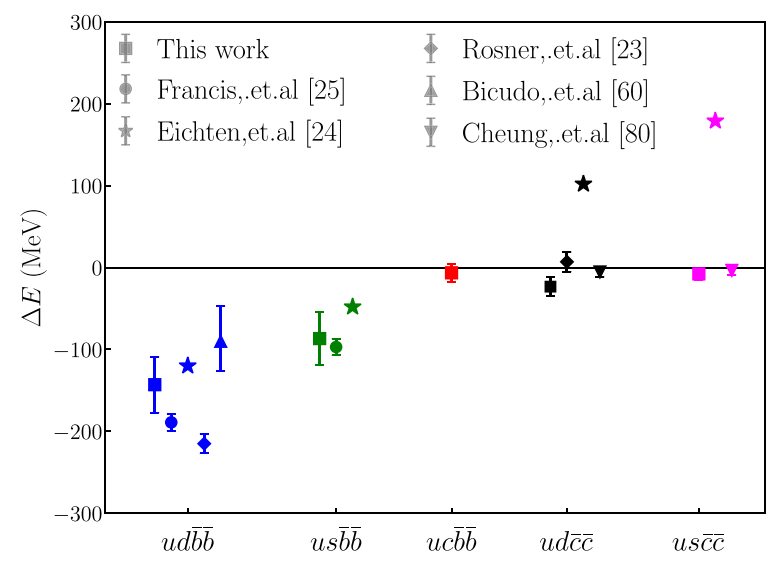

FIG. 9. Comparison of global results on the spin-1 doubly bottom and charm tetraquark states with various flavor combinations. $\Delta E$ is the energy difference between the ground state and the lowest strong decay threshold. Various flavor combinations represented on the horizontal axis are color coded as blue, green, red, magenta, and grey for the states $u d \bar{b} \bar{b}, u s \bar{b} \bar{b}, u c \bar{b} \bar{b}, u d \bar{c} \bar{c}$, and $u s \bar{c} \bar{c}$, respectively.

These calculations were performed at three pion masses ranging from $m_{\pi} \sim 340-650 \mathrm{MeV}$, and the final results were obtained after chiral extrapolation. Reference [80] used an anisotropic $N_{f}=2+1$ clover action, and results were obtained at a single lattice spacing $\left(a_{t} \sim 0.0035 \mathrm{fm}\right.$ with anisotropy 3.5) and at a single pion mass $\left(m_{\pi}=391 \mathrm{MeV}\right)$. For the doubly charm states, our results are in disagreement with those from the HQET results [24]. As we showed earlier, this discrepancy is due to the deviation of HQET relations at the charm quark mass.

Inspired by the results in the spin-1 sector, we also explore the spin-0 tetraquark states with doubly bottom as well as with doubly charm quarks. Here, we have computed flavor symmetric $u u \bar{b} \bar{b}$ and $u u \bar{c} \bar{c}$ states and also explored the pion mass dependence by dialing the light quark mass. To check the lattice spacing dependence of the observed results, we perform the calculation on three different lattice spacings. In addition, we have also computed the following flavor symmetric states, namely, $s s \bar{b} \bar{b}, s s \bar{c} \bar{c}$, and $c c \bar{b} \bar{b}$ at the physical strange, charm, and bottom quark masses. For the doubly bottom state $u u \bar{b} \bar{b}$, we find that the energy splittings $\left(\Delta E^{0}\right)$ are generally noisy and do not clearly exhibit a trend of increase in $\Delta E^{0}$ as the pion mass is lowered. Contrary to the results of its flavor antisymmetric cousin $u d \bar{b} \bar{b}$, the ground state energy of $u u \bar{b} \bar{b}$ coincides with its threshold at lower pion masses with no clear indication of any level below the threshold. For the doubly charm state, $u u \bar{c} \bar{c}$, the extracted energy levels clearly lie above their respective thresholds with no discernible dependence on pion mass, again contrary to the results of its flavor antisymmetric cousin $u d \bar{c} \bar{c}$. In performing the continuum extrapolation, no lattice spacing dependence is observed for the $u u \bar{b} \bar{b}$ state, while the $u u \bar{c} \bar{c}$ exhibits a mild
TABLE XI. Final results for the spin-0 tetraquarks.

\begin{tabular}{lccc}
\hline \hline State & $\Delta E^{\mathbf{0}}(\mathrm{MeV})$ & State & $\Delta E^{\mathbf{0}}(\mathrm{MeV})$ \\
\hline$u u \bar{b} \bar{b}$ & $-5(18)$ & $u u \bar{c} \bar{c}$ & $26(11)$ \\
$s s \bar{b} \bar{b}$ & $3(9)$ & $s s \bar{c} \bar{c}$ & $14(4)$ \\
$c c \bar{b} \bar{b}$ & $16(1)$ & & \\
\hline \hline
\end{tabular}

dependence on the lattice spacing. The flavor symmetric states $s s \bar{b} \bar{b}, s s \bar{c} \bar{c}$, and $c c \bar{b} \bar{b}$ exhibit similar qualitative features in that all the energy levels are found to be above their respective thresholds and no significant lattice spacing dependence is observed in the continuum extrapolation. Our final results for the spin-0 sector are shown in Table XI. In conclusion, the states in the spin- 0 sector do not indicate energy levels below their thresholds, suggesting it is very unlikely that there exists any doubly heavy bound tetraquark states with spin 0 .

The availability of energy values of spin-1 tetraquark states for a large number of light quark masses provide us an opportunity to investigate the mass relations [Eq. (17)] between different heavy flavored hadrons due to the heavy quark symmetry, as mentioned in Ref. [24]. For this, we redefine the relation as a ratio $(\mathcal{R})$ between different hadron masses [Eq. (18)] where a value of unity justifies the validity of such a mass relation, and any deviation from unity indicates the amount of breaking of the heavy quark symmetry at a given heavy quark mass. We find that for bottom quarks $\mathcal{R}_{b}=0.837(38)$, indicating that the bottom quark is very close to the heavy quark limit. On the contrary, at the charm quark mass, we find $\mathcal{R}_{c}=0.602(22)$, which substantially deviates from the heavy quark limit. This clearly suggests that the charm quark is not heavy enough to impose heavy quark symmetry relations among hadron masses such as in Eq. (17); i.e., as far those mass relations are concerned, one needs to be careful while treating the charm quark within HQET.

The tetraquark states studied in this work are computed in a single volume. In order to make conclusive statements about their scattering amplitudes and complex poles, one needs to carry out similar studies on multiple volumes followed by a finite volume analysis [41]. Such an analysis will especially be useful for the states which are close to their thresholds. However, a comprehensive finite volume analysis for a calculation that is reported here requires significantly large computational resources. Currently, that is beyond the scope of this work, but we intend to pursue such finite volume analysis in the near future. However, it is worth noting that the finite volume corrections for many heavy tetraquarks, particularly for which $\Delta E$ values are large, will be substantially suppressed. This is because, as has been pointed out before [81-83], such corrections to the observed energy splitting are suppressed not only because of its large value but also for the large masses of the threshold states, which are two heavy mesons in these 
cases. It is thus expected that such tetraquark states will be stable under strong interactions. Other errors related to our calculations, namely, unphysical sea quark mass, quark mass tuning, scale setting, mixed action effects, and excited state contamination, together will be much smaller compared to the statistical error [84], and the conclusion reached here will be unaffected by those. It will therefore be very useful to search experimentally spin-1 doubly heavy tetraquarks particularly with two bottom quarks, such as $u d \bar{b} \bar{b}$. However, it is very unlikely that there exists any doubly heavy bound tetraquark state with spin 0 .

\section{ACKNOWLEDGMENTS}

We are thankful to the MILC Collaboration and in particular to S. Gottlieb for providing us with the HISQ lattices. We would like to thank R. V. Gavai for discussions, particularly on HQET relations, and M. Hansen for the discussions on finite volume corrections. We also thank R. Lewis and M. Peardon for useful discussions. Computations were carried out on the Cray-XC30 of ILGTI, TIFR, and on the Gaggle/Pride clusters of the Department of Theoretical Physics, TIFR. P. J. and N. M. would like to thank Ajay Salve, Kapil Ghadiali, and P. M. Kulkarni for computational support. M. P. acknowledges support from the European Union under Grant No. MSCAIF-EF-ST-744659 (XQCDBaryons) and the Deutsche Forschungsgemeinschaft under Grant No. SFB/TRR 55.

\section{APPENDIX}

In Table XII, we show the energy splittings, $\Delta E$, defined as the difference between the threshold energy and the ground state energy levels, of tetraquark states with various flavor-spin combinations as studied in this work.

TABLE XII. Summary of splittings of tetraquark states in this work.

\begin{tabular}{|c|c|c|c|c|c|c|c|c|}
\hline$N_{s}^{3} \times N_{t}$ & $m_{\pi}(\mathrm{MeV})$ & $u d \bar{b} \bar{b}$ & $u s \bar{b} \bar{b}$ & $u c \bar{b} \bar{b}$ & $u d \bar{c} \bar{c}$ & $u s \bar{c} \bar{c}$ & $u u \bar{b} \bar{b}$ & $u u \bar{c} \bar{c}$ \\
\hline \multirow[t]{15}{*}{$24^{3} \times 64$} & 689 & $-83(9)$ & $-83(9)$ & $-15(4)$ & $-11(3)$ & $-11(3)$ & $17(10)$ & $11(2)$ \\
\hline & 589 & $-110(13)$ & $-101(9)$ & $-19(4)$ & $-18(3)$ & $-14(3)$ & $8(6)$ & $9(2)$ \\
\hline & 539 & $-117(16)$ & $-104(9)$ & $-22(4)$ & $-18(3)$ & $-12(2)$ & $6(6)$ & $11(2)$ \\
\hline & 497 & $-120(14)$ & $-100(14)$ & $-18(6)$ & $-22(5)$ & $-13(3)$ & $8(8)$ & $9(3)$ \\
\hline & 449 & $-127(18)$ & $-111(10)$ & $-25(5)$ & $-25(4)$ & $-13(2)$ & $4(10)$ & $10(3)$ \\
\hline & 400 & $-136(24)$ & $-111(12)$ & $-21(5)$ & $-27(5)$ & $-12(3)$ & $0(11)$ & $8(5)$ \\
\hline & 367 & $-145(21)$ & $-116(12)$ & $-29(5)$ & $-28(6)$ & $-12(3)$ & $-3(10)$ & $9(4)$ \\
\hline & 337 & $-146(25)$ & $-109(13)$ & $-20(8)$ & $-26(7)$ & $-11(3)$ & $-2(12)$ & $8(7)$ \\
\hline & 297 & $-164(36)$ & $-119(15)$ & $-30(6)$ & $-28(7)$ & $-11(3)$ & $\cdots$ & $6(7)$ \\
\hline & 257 & $-181(43)$ & $-115(18)$ & $-25(9)$ & $-25(8)$ & $-9(4)$ & $\cdots$ & $\ldots$ \\
\hline & 237 & $\ldots$ & $-112(21)$ & $-29(8)$ & $\cdots$ & $\ldots$ & $\cdots$ & $\cdots$ \\
\hline & 216 & $\ldots$ & $-117(14)$ & $-19(13)$ & $\ldots$ & $\ldots$ & $\ldots$ & $\ldots$ \\
\hline & 202 & $\ldots$ & $-126(18)$ & $-27(11)$ & $\ldots$ & $\ldots$ & $\cdots$ & $\ldots$ \\
\hline & 186 & $\ldots$ & $-121(17)$ & $-31(11)$ & $\ldots$ & $\ldots$ & $\ldots$ & $\ldots$ \\
\hline & 153 & $\ldots$ & $\ldots$ & $-33(13)$ & $\cdots$ & $\ldots$ & $\cdots$ & $\ldots$ \\
\hline \multirow[t]{7}{*}{$32^{3} \times 96$} & 688 & $-62(13)$ & $-62(13)$ & $-9(3)$ & $-13(3)$ & $-13(3)$ & $5(5)$ & $12(3)$ \\
\hline & 537 & $-93(19)$ & $-77(15)$ & $-12(5)$ & $-19(5)$ & $-13(3)$ & $-1(8)$ & $9(7)$ \\
\hline & 491 & $-123(25)$ & $-74(23)$ & $-14(5)$ & $-23(6)$ & $-14(4)$ & $-2(10)$ & 12(9) \\
\hline & 441 & $-135(21)$ & $-79(18)$ & $-12(5)$ & $-23(8)$ & $-10(4)$ & $-6(12)$ & $15(12)$ \\
\hline & 396 & $-147(31)$ & $-91(17)$ & $-16(5)$ & $-27(10)$ & $-10(5)$ & $-6(13)$ & $19(8)$ \\
\hline & 367 & $\ldots$ & $-97(19)$ & $-15(6)$ & $-32(13)$ & $-9(5)$ & $0(11)$ & $\ldots$ \\
\hline & 345 & $\cdots$ & $\ldots$ & $-17(6)$ & $\ldots$ & $\ldots$ & $-5(13)$ & $\ldots$ \\
\hline \multirow[t]{4}{*}{$48^{3} \times 144$} & 685 & $-88(6)$ & $-88(6)$ & $-10(2)$ & $-15(2)$ & $-15(2)$ & $6(7)$ & $17(2)$ \\
\hline & 645 & $-94(7)$ & $-91(7)$ & $-11(2)$ & $-15(2)$ & $-13(3)$ & $4(7)$ & $17(3)$ \\
\hline & 576 & $-102(9)$ & $-94(8)$ & $-10(2)$ & $-15(3)$ & $-13(2)$ & $3(8)$ & $18(4)$ \\
\hline & 545 & $-106(10)$ & $-90(10)$ & $-12(3)$ & $-17(3)$ & $-13(3)$ & $-1(8)$ & $20(4)$ \\
\hline
\end{tabular}


[1] S. Dobbs et al. (CLEO Collaboration), Precision Measurement of the Mass of the $h_{c}\left({ }^{1} P_{1}\right)$ State of Charmonium, Phys. Rev. Lett. 101, 182003 (2008).

[2] M. Ablikim et al. (BESIII Collaboration), Measurements of $h_{c}\left({ }^{1} P_{1}\right)$ in $\psi^{\prime}$ Decays, Phys. Rev. Lett. 104, 132002 (2010).

[3] I. Adachi et al. (Belle Collaboration), First Observation of the $P$-Wave Spin-Singlet Bottomonium States $h_{b}(1 P)$ and $h_{b}(2 P)$, Phys. Rev. Lett. 108, 032001 (2012).

[4] J. P. Lees et al. (BABAR Collaboration), Evidence for the $h_{b}(1 P)$ meson in the decay $\Upsilon(3 S) \rightarrow \pi^{0} h_{b}(1 P)$, Phys. Rev. D 84, 091101 (2011).

[5] M. Ablikim et al. (BESIII Collaboration), Observation of the $\psi\left(1^{3} D_{2}\right)$ State in $e^{+} e^{-} \rightarrow \pi^{+} \pi^{-} \gamma \chi_{c 1}$ at BESIII, Phys. Rev. Lett. 115, 011803 (2015).

[6] V. Bhardwaj et al. (Belle Collaboration), Evidence of a New Narrow Resonance Decaying to $\chi_{c 1} \gamma$ in $B \rightarrow \chi_{c 1} \gamma K$, Phys. Rev. Lett. 111, 032001 (2013).

[7] A. M. Sirunyan et al. (CMS Collaboration), Observation of the $\chi_{\mathrm{b} 1}(3 \mathrm{P})$ and $\chi_{\mathrm{b} 2}(3 \mathrm{P})$ and Measurement of their Masses, Phys. Rev. Lett. 121, 092002 (2018).

[8] R. Aaij et al. (LHCb Collaboration), Observation of the Doubly Charmed Baryon $\Xi_{c c}^{++}$, Phys. Rev. Lett. 119, 112001 (2017).

[9] R. Aaij et al. (LHCb Collaboration), Observation of a New $\Xi_{b}^{-}$Resonance, Phys. Rev. Lett. 121, 072002 (2018).

[10] S. K. Choi et al. (Belle Collaboration), Observation of a Resonance-Like Structure In the $\pi^{+-} \psi^{\prime}$ Mass Distribution in Exclusive $B \rightarrow K \pi^{+-} \psi^{\prime}$ Decays, Phys. Rev. Lett. 100, 142001 (2008).

[11] R. Aaij et al. (LHCb Collaboration), Observation of the Resonant Character of the $Z(4430)^{-}$State, Phys. Rev. Lett. 112, 222002 (2014).

[12] A. Bondar et al. (Belle Collaboration), Observation of Two Charged Bottomonium-Like Resonances in Y(5S) Decays, Phys. Rev. Lett. 108, 122001 (2012).

[13] R. Aaij et al. (LHCb Collaboration), Observation of $J / \psi p$ Resonances Consistent with Pentaquark States in $\Lambda_{b}^{0} \rightarrow J / \psi K^{-} p$ Decays, Phys. Rev. Lett. 115, 072001 (2015).

[14] S. K. Choi et al. (Belle Collaboration), Observation of a Narrow Charmonium-Like State in Exclusive $B^{ \pm} \rightarrow$ $K^{ \pm} \pi^{+} \pi^{-} J / \psi$ Decays, Phys. Rev. Lett. 91, 262001 (2003).

[15] B. Aubert et al. (BABAR Collaboration), Observation of a Broad Structure in the $\pi^{+} \pi^{-} J / \psi$ Mass Spectrum around 4.26-GeV/c², Phys. Rev. Lett. 95, 142001 (2005).

[16] C.Z. Yuan et al. (Belle Collaboration), Measurement of $e^{+} e^{-} \rightarrow \pi^{+} \pi^{-} J / \psi$ Cross-Section via Initial State Radiation at Belle, Phys. Rev. Lett. 99, 182004 (2007).

[17] M. B. Voloshin, Charmonium, Prog. Part. Nucl. Phys. 61, 455 (2008).

[18] H.-X. Chen, W. Chen, X. Liu, and S.-L. Zhu, The hiddencharm pentaquark and tetraquark states, Phys. Rep. 639, 1 (2016).

[19] A. Hosaka, T. Iijima, K. Miyabayashi, Y. Sakai, and S. Yasui, Exotic hadrons with heavy flavors: X, Y, Z, and related states, Prog. Theor. Exp. Phys. 2016, 062C01 (2016).
[20] A. Ali, J. S. Lange, and S. Stone, Exotics: Heavy pentaquarks and tetraquarks, Prog. Part. Nucl. Phys. 97, 123 (2017).

[21] R. Aaij et al. (LHCb Collaboration), Determination of the X (3872) Meson Quantum Numbers, Phys. Rev. Lett. 110, 222001 (2013).

[22] M. Tanabashi et al. (Particle Data Group), Review of particle physics, Phys. Rev. D 98, 030001 (2018).

[23] M. Karliner and J. L. Rosner, Discovery of DoublyCharmed $\Xi_{c c}$ Baryon Implies a Stable $(b b \bar{u} \bar{d})$ Tetraquark, Phys. Rev. Lett. 119, 202001 (2017).

[24] E. J. Eichten and C. Quigg, Heavy-Quark Symmetry Implies Stable Heavy Tetraquark Mesons $Q_{i} Q_{j} \bar{q}_{k} \bar{q}_{l}$, Phys. Rev. Lett. 119, 202002 (2017).

[25] A. Francis, R. J. Hudspith, R. Lewis, and K. Maltman, Lattice Prediction for Deeply Bound Doubly Heavy Tetraquarks, Phys. Rev. Lett. 118, 142001 (2017).

[26] A. Ali, Q. Qin, and W. Wang, Discovery potential of stable and near-threshold doubly heavy tetraquarks at the LHC, Phys. Lett. B 785, 605 (2018).

[27] A. Esposito, A. Pilloni, and A. D. Polosa, Multiquark resonances, Phys. Rep. 668, 1 (2017).

[28] R. F. Lebed, R. E. Mitchell, and E. S. Swanson, Heavy-quark QCD exotica, Prog. Part. Nucl. Phys. 93, 143 (2017).

[29] S. L. Olsen, T. Skwarnicki, and D. Zieminska, Nonstandard heavy mesons and baryons: Experimental evidence, Rev. Mod. Phys. 90, 015003 (2018).

[30] R. L. Jaffe, Multi-quark hadrons. 1. The phenomenology of (2 quark 2 anti-quark) mesons, Phys. Rev. D 15, 267 (1977).

[31] R. Jaffe, Exotica, Phys. Rep. 409, 1 (2005).

[32] R. L. Jaffe and F. Wilczek, Diquarks and Exotic Spectroscopy, Phys. Rev. Lett. 91, 232003 (2003).

[33] E. Braaten and M. Kusunoki, Low-energy universality and the new charmonium resonance at $3870-\mathrm{MeV}$, Phys. Rev. D 69, 074005 (2004).

[34] F.-K. Guo, C. Hanhart, U.-G. Meisner, Q. Wang, Q. Zhao, and B.-S. Zou, Hadronic molecules, Rev. Mod. Phys. 90, 015004 (2018).

[35] S. Dubynskiy and M. B. Voloshin, Hadro-Charmonium, Phys. Lett. B 666, 344 (2008).

[36] E. Braaten, How the $Z_{c}(3900)$ Reveals the Spectra of Quarkonium Hybrid and Tetraquark Mesons, Phys. Rev. Lett. 111, 162003 (2013).

[37] N. A. Tornqvist, Understanding the scalar meson q anti-q nonet, Z. Phys. C 68, 647 (1995).

[38] D. V. Bugg, How resonances can synchronise with thresholds, J. Phys. G 35, 075005 (2008).

[39] M. Peardon, J. Bulava, J. Foley, C. Morningstar, J. Dudek, R. G. Edwards, B. Joo, H.-W. Lin, D. G. Richards, and K. J. Juge (Hadron Spectrum Collaboration), A novel quark-field creation operator construction for hadronic physics in lattice QCD, Phys. Rev. D 80, 054506 (2009).

[40] C. Michael, Adjoint sources in lattice gauge theory, Nucl. Phys. B259, 58 (1985).

[41] M. Luscher and U. Wolff, How to calculate the elastic scattering matrix in two-dimensional quantum field theories by numerical simulation, Nucl. Phys. B339, 222 (1990). 
[42] A. V. Manohar and M. B. Wise, Exotic Q Q anti-q anti-q states in QCD, Nucl. Phys. B399, 17 (1993).

[43] T. Mehen, Implications of heavy quark-diquark symmetry for excited doubly heavy baryons and tetraquarks, Phys. Rev. D 96, 094028 (2017).

[44] L. Maiani, F. Piccinini, A. D. Polosa, and V. Riquer, Diquark-antidiquarks with hidden or open charm and the nature of X(3872), Phys. Rev. D 71, 014028 (2005).

[45] Y. Cui, X.-L. Chen, W.-Z. Deng, and S.-L. Zhu, The Possible Heavy Tetraquarks $q Q \bar{q} \bar{Q}, q q \bar{Q} \bar{Q}$ and $q Q \bar{Q} \bar{Q}$, High Energy Phys. Nucl. Phys. 31, 7 (2007).

[46] D. Ebert, R. N. Faustov, V. O. Galkin, and W. Lucha, Masses of tetraquarks with two heavy quarks in the relativistic quark model, Phys. Rev. D 76, 114015 (2007).

[47] D. Ebert, R. N. Faustov, and V. O. Galkin, Excited heavy tetraquarks with hidden charm, Eur. Phys. J. C 58, 399 (2008).

[48] A. Ali, L. Maiani, A. D. Polosa, and V. Riquer, Hiddenbeauty charged tetraquarks and heavy quark spin conservation, Phys. Rev. D 91, 017502 (2015).

[49] J.-M. Richard, A. Valcarce, and J. Vijande, Few-body quark dynamics for doubly heavy baryons and tetraquarks, Phys. Rev. C 97, 035211 (2018).

[50] W. Wilcox and S. Baral, Multiquark states in the ThomasFermi quark model and on the lattice, arXiv:1807.08626.

[51] Z.-G. Wang, Y.-M. Xu, and H.-J. Wang, Analysis of the scalar doubly heavy tetraquark states with QCD sum rules, Commun. Theor. Phys. 55, 1049 (2011).

[52] Z.-G. Wang and T. Huang, Analysis of the $X(3872)$, $Z_{c}(3900)$ and $Z_{c}(3885)$ as axial-vector tetraquark states with QCD sum rules, Phys. Rev. D 89, 054019 (2014).

[53] R. T. Kleiv, T. G. Steele, A. Zhang, and I. Blokland, Heavylight diquark masses from QCD sum rules and constituent diquark models of tetraquarks, Phys. Rev. D 87, 125018 (2013).

[54] S. Weinberg, Tetraquark Mesons in Large $N$ Quantum Chromodynamics, Phys. Rev. Lett. 110, 261601 (2013).

[55] M. Knecht and S. Peris, Narrow Tetraquarks at Large N, Phys. Rev. D 88, 036016 (2013).

[56] T. D. Cohen and R. F. Lebed, Are there tetraquarks at large $N_{c}$ in QCD(F)? Phys. Rev. D 90, 016001 (2014).

[57] P. Junnarkar, M. Padmanath, and N. Mathur, Heavy light tetraquarks from Lattice QCD, EPJ Web Conf. 175, 05014 (2018).

[58] P. Bicudo, J. Scheunert, and M. Wagner, Including heavy spin effects in the prediction of a $\bar{b} \bar{b} u d$ tetraquark with lattice QCD potentials, Phys. Rev. D 95, 034502 (2017).

[59] P. Bicudo, K. Cichy, A. Peters, B. Wagenbach, and M. Wagner, Evidence for the existence of $u d \bar{b} \bar{b}$ and the nonexistence of $s s \bar{b} \bar{b}$ and $c c \bar{b} \bar{b}$ tetraquarks from lattice QCD, Phys. Rev. D 92, 014507 (2015).

[60] P. Bicudo, K. Cichy, A. Peters, and M. Wagner, BB interactions with static bottom quarks from Lattice QCD, Phys. Rev. D 93, 034501 (2016).

[61] A. Bazavov et al. (MILC Collaboration), Lattice QCD ensembles with four flavors of highly improved staggered quarks, Phys. Rev. D 87, 054505 (2013).

[62] E. Follana, Q. Mason, C. Davies, K. Hornbostel, G. P. Lepage, J. Shigemitsu, H. Trottier, and K. Wong (HPQCD and UKQCD Collaboration), Highly improved staggered quarks on the lattice, with applications to charm physics, Phys. Rev. D 75, 054502 (2007).

[63] H. Neuberger, Exactly massless quarks on the lattice, Phys. Lett. B 417, 141 (1998).

[64] H. Neuberger, More about exactly massless quarks on the lattice, Phys. Lett. B 427, 353 (1998).

[65] M. Luscher, Exact chiral symmetry on the lattice and the Ginsparg-Wilson relation, Phys. Lett. B 428, 342 (1998).

[66] Y. Chen, S. J. Dong, T. Draper, I. Horvath, F. X. Lee, K. F. Liu, N. Mathur, and J. B. Zhang, Chiral logarithms in quenched QCD, Phys. Rev. D 70, 034502 (2004).

[67] A. Li et al. (XQCD Collaboration), Overlap valence on $2+1$ flavor domain wall fermion configurations with deflation and low-mode substitution, Phys. Rev. D 82, 114501 (2010).

[68] B. Chakraborty, C. T. H. Davies, B. Galloway, P. Knecht, J. Koponen, G. C. Donald, R. J. Dowdall, G. P. Lepage, and C. McNeile, High-precision quark masses and QCD coupling from $n_{f}=4$ lattice QCD, Phys. Rev. D 91, 054508 (2015).

[69] S. Basak, S. Datta, M. Padmanath, P. Majumdar, and N. Mathur, Charm and strange hadron spectra from overlap fermions on HISQ gauge configurations, Proc. Sci. LATTICE2012 (2012) 141 [arXiv:1211.6277].

[70] S. Basak, S. Datta, A. T. Lytle, M. Padmanath, P. Majumdar, and N. Mathur, Hadron spectra from overlap fermions on HISQ gauge configurations, Proc. Sci. LATTICE2013 (2014) 243 [arXiv:1312.3050].

[71] A. X. El-Khadra, A. S. Kronfeld, and P. B. Mackenzie, Massive fermions in lattice gauge theory, Phys. Rev. D 55, 3933 (1997).

[72] G. P. Lepage, L. Magnea, C. Nakhleh, U. Magnea, and K. Hornbostel, Improved nonrelativistic QCD for heavy quark physics, Phys. Rev. D 46, 4052 (1992).

[73] R. Lewis and R. M. Woloshyn, Bottom baryons from a dynamical lattice QCD simulation, Phys. Rev. D 79, 014502 (2009).

[74] R. J. Dowdall et al. (HPQCD Collaboration), The Upsilon spectrum and the determination of the lattice spacing from lattice QCD including charm quarks in the sea, Phys. Rev. D 85, 054509 (2012).

[75] N. Mathur, M. Padmanath, and R. Lewis, Charmed-bottom mesons from lattice QCD, Proc. Sci. LATTICE2016 (2016) 100. [arXiv:1611.04085].

[76] M. Padmanath, C. B. Lang, and S. Prelovsek, X(3872) and $\mathrm{Y}(4140)$ using diquark-antidiquark operators with lattice QCD, Phys. Rev. D 92, 034501 (2015).

[77] B. Blossier, M. Della Morte, G. von Hippel, T. Mendes, and R. Sommer, On the generalized eigenvalue method for energies and matrix elements in lattice field theory, J. High Energy Phys. 04 (2009) 094.

[78] J. Green, A. Francis, P. Junnarkar, C. Miao, T. Rae, and H. Wittig, Search for a bound H-dibaryon using local six-quark interpolating operators, Proc. Sci. LATTICE2014 (2014) 107 [arXiv:1411.1643].

[79] A. Francis, J. R. Green, P. M. Junnarkar, C. Miao, T. D. Rae, and H. Wittig, Lattice QCD study of the $H$ dibaryon using hexaquark and two-baryon interpolators, arXiv:1805 .03966

[80] G. K. C. Cheung, C. E. Thomas, J. J. Dudek, and R. G. Edwards (Hadron Spectrum Collaboration), Tetraquark 
operators in lattice QCD and exotic flavour states in the charm sector, J. High Energy Phys. 11 (2017) 033.

[81] S. R. Beane, P. F. Bedaque, A. Parreno, and M. J. Savage, Two nucleons on a lattice, Phys. Lett. B 585, 106 (2004).

[82] Z. Davoudi and M. J. Savage, Improving the volume dependence of two-body binding energies calculated with lattice QCD, Phys. Rev. D 84, 114502 (2011).
[83] R. A. Briceño, Z. Davoudi, T. Luu, and M. J. Savage, Two-nucleon systems in a finite volume. II. ${ }^{3} S_{1}-{ }^{3} D_{1}$ coupled channels and the deuteron, Phys. Rev. D 88, 114507 (2013).

[84] N. Mathur, M. Padmanath, and S. Mondal, Precise predictions of charmed-bottom hadrons from lattice QCD, Phys. Rev. Lett. 121, 202002 (2018). 